COMECHINGONIA. Revista de Arqueología. Vol. 22, n² 1. Primer semestre de 2018. ISSN: 0326-7911

COMITÉ EDITORIAL

DIRECTOR

SEBASTIÁN PASTOR (CITCA-CONICET-Catamarca)

CO-EDITORA

ANDREA RECALDE (CEH-IEH-CONICET-UNC-Córdoba)

CONSEJO ASESOR

JESÚS ADÁNEZ PAVÓN (UNIVERSIDAD COMPLUTENSE-Madrid)

J. ROBERTO BÁRCENA (INCIHUSA-CONICET-UNCU-Mendoza)

LUIS F. BATE (ENAH-México)

LUIS BORRERO (IMHICIHU-CONICET-Buenos Aires)

FELIPE CRIADO BOADO (INCIPIT-CSIC-Santiago de Compostela)

LEONARDO GARCÍA SANJÚAN (U. DE SEVILLA-Sevilla)

GUILLERMO MENGONI GOÑALONS (ICA-UBA-CONICET-Buenos Aires)

AXEL NIELSEN (INAPL-CONICET-Buenos Aires)

GUSTAVO POLITIS (INCUAPA-CONICET-UNCPB-Olavarría)

MYRIAM TARRAGÓ (M. ETNOGRÁFICO-UBA-CONICET-Buenos Aires)

HUGO YACOBACCIO (ICA-UBA-CONICET-Buenos Aires)

EVALUADORES PARA ESTE NÚMERO

Federico Bobillo (ISES-CONICET); María F. Bugliani (IDECU-UBA-CONICET); Rafael

Curtoni (INCUAPA-CONICET-UNCPBA); Violeta Di Prado (FCNyM-UNLP-CONICET);

Anabel Feely (IDECU-UBA-CONICET); Fernanda Falabella (Dpto. de Antropología, Universidad de Chile); Emilia Iucci (FCNyM-UNLP-CONICET ); Jimena Oria (CADICCONICET); María C. Páez (CONICET-FCNyM-UNLP); Valeria Palamarzuck (IDECUUBA-CONICET); Rafael Paunero (FCNyM-UNLP); José Porto López (LARBO-UNMDP); Cristina Prieto Olavarría (IANIGLA-CONICET); Federico Restifo (CONICET-FFyH-UBA);

Camila Riera Soto (Universidad Católica del Norte, Chile); Julián Salazar (CEH-IEHCONICET-UNC); M. Lorena Sanhueza Riquelme (Dpto. de Antropología, Universidad de Chile); Marina Sprovieri (CONICET-FCNyM-UNLP); Emily Stovel (El Zócalo Plaza Visitor and Event Center, Sandoval County Office of Economic Development, Bernalillo); Nuria Sugrañes (IANIGLA-CONICET); Veronika Szilágyi (Nuclear Analysis and Radiography Department, Centre for Energy Research, Hungarian Academy of Sciences); Cristian Vitry (UNSa); Verónica Williams (IDECU-UBA-CONICET); Federico Wynveldt (CONICETFCNyM-UNLP); M. Amalia Zaburlin (CREA-FHyCS-UNJu).

Dirección postal: Miguel C. del Corro 308, (5000) Córdoba - Argentina

Correo electrónico: revistacomechingonia@gmail.com

Web: http//www.comechingonia.com 

COMECHINGONIA. Revista de Arqueología. Vol. 22, n 1. Primer semestre de 2018. ISSN: 0326-7911

\section{Índice}

Presentación

Dossier: "Problemáticas y abordajes metodológicos en los estudios petrográficos de cerámica arqueológica".

1. Presentación.

Por: Lucas Pereyra Domingorena y Verónica Puente

2. Un volcán en la cerámica: indicios de producción en el altiplano de Isluga,

Tarapacá-Chile.

Por: Isabelle Druc y Mauricio Uribe Rodríguez

3. Una puesta al día sobre la variación petrográfica y química del estilo cerámico Inca Pacajes.

Por: Nicolás Larcher y M. Beatriz Cremonte

4. Elecciones tecnológicas y prácticas alfareras en el tambo Costa de Reyes $\mathrm{N}^{\mathrm{o}}$

5 (Tinogasta, Catamarca): aproximaciones petrográficas.

Por: Sergio Vera y Guillermo de la Fuente

5. Tecnología cerámica a través de la petrografía en la Quebrada de Miriguaca (Antofagasta de la Sierra, Catamarca) durante el Período Tardío.

Por: Leticia Gasparotti

6. Nuevas evidencias en relación a la selección de materiales cerámicos para

formar parte de los contextos funerarios. PP9-III y PP13-I como casos de estudio (ca. 1330-1000 AP) (Antofagasta de la Sierra, Catamarca).

Por: Vanesa Juárez

7. Prácticas de manufactura cerámica en el oeste riojano durante el Período

Tardío (ca. siglos XIII-XVII AD). Aportes desde los estudios petrográficos sobre el estilo Sanagasta/ Angualasto de la Tambería de Guandacol.

Por: Sebastián Carosio

8. Evidencias arqueológicas de los siglos VII a XII AD en el asentamiento de Moreta (Puna de Jujuy, Argentina).

Por: M. Josefina Pérez Pieroni y Carlos Angiorama

9. Una aproximación petrográfica a los modos de hacer y la circulación alfarera en el Área Valliserrana del Noroeste Argentino durante los últimos siglos del primer milenio D.C.

Por: Lucas Pereyra Domingorena y Verónica Puente

\section{Artículos}

1. Nuevos datos en relación a la arqueología del valle de Lerma, provincia de

Salta, Argentina.

Por: Cecilia Mercuri

2. Aplicación de los drones en diversos contextos arqueológicos y en casos de reconstrucción geoarqueológica.

Por: M. Marta Sampietro Vattuone y José Peña Monné 
3. Ritualidad y espacialidad andina a través de enfoques etnográficos y arqueológicos. Confluencias cosmogónico-ontológicas en la constitución de espacios sagrados entre El Shincal de Quimivil y ceremonias actuales de origen cusqueño.

Por: Marco Giovannetti

4. El paisaje rupestre de Cerro Colorado (provincia de Córdoba, Argentina): detectando otros sentidos, otras corporalidades.

Por: Luis Tissera 
COMECHINGONIA. Revista de Arqueología. Vol. 22, n 1. Primer semestre de 2018. ISSN: 0326-7911

\title{
UNA APROXIMACIÓN PETROGRÁFICA A LOS MODOS DE HACER Y LA CIRCULACIÓN ALFARERA EN EL ÁREA VALLISERRANA DEL NOROESTE ARGENTINO DURANTE LOS ÚLTIMOS SIGLOS DEL PRIMER MILENIO D.C. \\ A PETROGRAPHICAL APPROACH TO THE WAYS OF DOING AND THE POTTERY CIRCULATION IN THE VALLEYS \& HILLS AREA OF THE ARGENTINE NORTHWEST DURING THE LAST CENTURIES OF THE FIRST MILLENIUM AD
}

\author{
Lucas Pereyra Domingorena ${ }^{1}$ y Verónica Puente ${ }^{2}$ \\ ${ }^{1}$ IDECU, UBA - CONICET. Moreno 350, (1091) Buenos Aires, Argentina, \\ lucasdomingorena@gmail.com; \\ 2 CONICET, LARBO - UNMDP. Juan B. Justo 2550, (7600) Mar del Plata, Argentina, \\ vpuente78@yahoo.com.ar
}

Presentado: 27/09/2017 - Aceptado: 12/06/2018

\section{Resumen}

Esta investigación procura indagar sobre los modos de hacer cerámica que tenían las sociedades prehispánicas de finales del primer milenio D.C. que habitaron el sur de los valles Calchaquíes y los valles de El Bolsón y Hualfin en el Noroeste argentino. Las pastas de los tiestos seleccionados fueron analizadas petrográficamente, utilizando una metodología que describe las estructuras de fondo de la matriz arcillosa y la identificación, cuantificación y registro de las inclusiones no plásticas y de las cavidades. El análisis de estas variables en conjunto, permitió caracterizar las elecciones técnicas realizadas por las alfareras y alfareros durante las etapas de la elaboración de las piezas, e identificar la existencia de patrones de manufactura. Asimismo, la determinación de la naturaleza de los temperantes fue fundamental para vincular piezas de posible procedencia homóloga. El carácter macrorregional de la muestra posibilitó detectar circulación de ciertas piezas y regularidades de manufactura que permiten postular la presencia de un savoir faire de confección cerámica, es decir, modos de hacer vasijas con recurrencias a escalas mayores y particularidades locales al interior de estos modos.

Palabras clave: materias primas, producción, intercambio, modos de hacer

\begin{abstract}
This work argues about 'the ways of doing pottery' that pre-Hispanic societies had at the end of the first millennium A.D. in the southern of Calchaqui valley and the El Bolsón and Hualfin valleys (Northwestern Argentina). The petrography of ceramic pastes was analyzed using a methodology that describes the structures of the clay matrix and the identification, quantification and registration
\end{abstract}


of non-plastic inclusions and of cavities. Also, the determination of the nature of the tempering was essential to link pieces of possible homologous origin. The macro-regional character of the sample made it possible to detect circulation of the certain pieces and manufacturing regularities and postulate the presence of a ceramics manufacturing savoir faire, that is, ways of making vessels with recurrences to larger scales and local peculiarities within these modes.

Keywords: raw materials, production, exchange, ways of doing

\section{Problemática y objetivos de la investigación}

La evidencia más antigua de cerámica en el Noroeste argentino data del 3000 AP aproximadamente, relacionada con sociedades cazadoras-recolectoras en transición hacia una economía agro-pastoril (García 1997; Cremonte et al. 2010). Desde entonces y hasta al menos los primeros años del Periodo Colonial, la alfarería fue una de las principales producciones artesanales que participó en diversas prácticas cotidianas, tanto en la cocción y el servicio de alimentos como en el almacenaje de recursos. Pero también formó parte de los contextos funerarios como urna y/o acompañamiento mortuorio. Las escalas y formas de organización de la producción fueron diversas y más allá de sus propiedades particulares, las vasijas se utilizaron como soporte de representaciones iconográficas cargadas de significación para quienes las elaboraron y consumieron. Además, tanto como objeto terminado así como durante su proceso de producción, fue un medio a través del cual se construyeron y reprodujeron relaciones sociales de distinta índole. En esta diversidad de contextos y prácticas de interacción, las piezas y los conocimientos sobre cómo elaborarlas circularon a distintas escalas.

En relación con ello, el tema de investigación de este trabajo es el estudio de las prácticas de producción alfarera que coexistieron durante los últimos siglos del primer milenio D.C. en parte de la región Valliserrana del Noroeste argentino. En ese momento, en las sociedades agropastoriles comienza un proceso de reorganización del espacio social caracterizado por la separación de las áreas productivas de las residenciales (Scatollin 2006a). Respecto a la cerámica se empiezan a registrar nuevas modalidades morfológicas -ollas de cuerpo globular con cuello cilíndrico corto- y mayor inversión de trabajo en los tratamientos de superficie y representaciones iconográficas, que posiblemente estuvieran vinculadas al "incremento del uso de bebidas en ciertos contextos de consumo social" (Scatollin 2007a: 302).

En ese contexto de cambios sociopolíticos, coexistieron distintas formas de interacción a distancia que podrían estar representadas en los modelos de tráfico caravanero (Núñez y Dillehay 1979), tráfico incorporado (Nielsen 2006) y relaciones interfamiliares (Aschero 
2007). Sin poder determinar la modalidad específica de interacción, se reconoce en el Noroeste argentino la circulación de bienes y materias primas tales como cerámicas, obsidianas, arcillas, maderas, etc. (Lazzari 2006; Aschero 2007; Olivera et al. 2015; Lazzari et al. 2017, entre otros). Además, la circulación de saberes a escala macrorregional se manifiesta en una estética compartida respecto a las siluetas de las vasijas y los recursos plásticos utilizados, tales como el color, la pintura, la incisión y/o el pulido (Scatollin 2007a).

Dentro del estudio de las prácticas de producción alfarera, el propósito de este artículo es determinar la existencia de tradiciones de manufactura compartidas o diferenciadas entre los habitantes de la región valliserrana y discutir la circulación de vasijas y saberes sobre cómo realizarlas. Esto implica por un lado, definir modalidades de elaboración y determinar si hay una tendencia geográfica en su distribución, y por el otro, analizar la relación entre éstas y la composición de las pastas con la finalidad de acercarnos a las regiones de producción, indagando además sobre cómo estos aspectos se vinculan con las características estilísticas de las piezas.

Estos objetivos son abordados a partir de los estudios petrográficos. Esta herramienta analítica aplicada sobre cerámica arqueológica permite obtener información significativa sobre las características composicionales del material antiplástico y los modos de preparación de las pastas, como indicadores de las primeras etapas del proceso de manufactura (Middleton y Freestone 1991; Cremonte 1996, 2014; Druc y Gwyn 1998; Stoltman 2001; Pereyra Domingorena 2010; Puente 2012, entre otros). Los aportes al respecto están representados en numerosos trabajos de índole arqueológica y etnoarqueológica y su aplicación en Sudamérica se ha incrementado notablemente en los últimos años.

\section{Área de estudio y cronología}

El conjunto estudiado integra, desde una perspectiva macrorregional, información generada de manera independiente por los autores para sus investigaciones previas. El mismo, procede de un área geográfica comprendida por los valles de El Bolsón, Hualfín y el sur Calchaquí, esta última conformada por la Falda occidental del Aconquija y el valle de Santa María. Los tiestos analizados provienen de los sitios arqueológicos de Tolombón, Bañado Viejo, Masao, Caspinchango-Mesa Redonda y Ampajango (llanura aluvional del río Santa María); Morro de las Espinillas, Tesoro I y Loma Alta (faldeo occidental del Aconquija); Los Viscos (valle de El Bolsón) y La Ciénaga (valle de Hualfín) (Figura 1). Estos

yacimientos fueron intervenidos a través de recolecciones superficiales y excavaciones sistemáticas y poseen distintas características contextuales y constructivas. Los casos estudiados se corresponden al rango cronológico que se extiende entre ca. 650-1000 D.C. (Tabla 1). 
El sitio Bañado Viejo se encuentra a unos 3,5 km al norte de la actual localidad de El Bañado (Provincia de Tucumán) y los hallazgos proceden de una excavación realizada en el valle aluvional del río Santa María. La coherencia cronológica de los materiales arqueológicos contenidos en la secuencia sedimentaria fue confirmada por el orden de las fechas radiocarbónicas, los resultados del análisis tafonómico de los restos óseos y la distribución tanto de los estilos cerámicos como los artefactos líticos asociados (Scattolin et al. 2001). Esta secuencia estratigráfica ha permitido distinguir tres bloques cronológicos y los tiestos aquí estudiados proceden del componente superior con un fechado asociado de $1170 \pm 40$ AP (Scattolin 2007b).

Los materiales procedentes de Caspinchango-Mesa Redonda (andén de cultivo), Masao y Ampajango son cerámicas recolectadas superficialmente en las campañas arqueológicas realizadas por la Universidad Nacional del Litoral a finales de la década de 1950 (Cigliano 1960; Tarrago y Scattolin 1999). Los fragmentos analizados de Tolombón fueron hallados en las cercanías de dicha localidad por la expedición arqueológica dirigida Ciro R. Lafón y Pedro Krapovickas, durante el año 1956 (Catálogo N¹2, actualmente se encuentra depositado en el Museo Etnográfico "Juan B. Ambrossetti" de la FFyL-UBA). Estas recolecciones superficiales han sido adscritas al rango cronológico estudiado por los diseños plásticos plasmados en sus superficies externas.

El sitio Morro de las Espinillas se encuentra a $2 \mathrm{~km}$ al sureste de la localidad de Punta de Balasto (Provincia de Catamarca) y presenta un patrón arquitectónico conglomerado, asignable al Período de Desarrollos Regionales pero relacionado con cerámica atribuida a los siglos finales del período Formativo (Scattolin 2010). Se cuenta con dos fechados

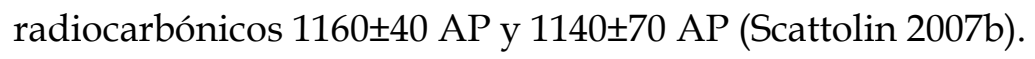

El sitio Tesoro I se localiza en las estribaciones de las Sierras del Aconquija, sobre la margen derecha del río Tesoro y está compuesto de unas 60 estructuras de canchones de cultivo y corrales (Scattolin y Albeck 1994). Se detectaron varios núcleos habitacionales pequeños que fueron sondeados en distintos trabajos de campo (Lazzari 2006). La muestra cerámica analizada proviene de las excavaciones realizadas en la Estructura Sur del Núcleo

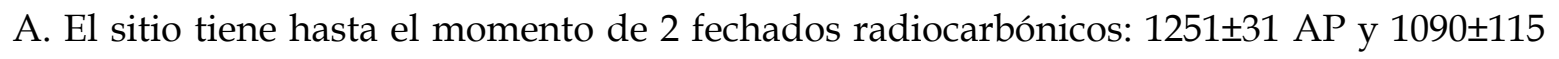
AP (Scattolin 2007b).

Loma Alta es un sitio arqueológico conformado por unas 100 estructuras mayormente subcirculares y de tamaños diversos. Entre éstas se han detectado nueve núcleos habitacionales intercalados entre campos de cultivos adosados a ellos (Scattolin 2010). Se seleccionó para esta muestra los materiales provenientes de las excavaciones realizadas en los núcleos habitacionales A, F y G correspondientes a la ocupación de finales del primer 
milenio d.C. Dicha ocupación se corrobora por los fechados radiocarbónicos y las características de los diseños plásticos de la cerámica. Actualmente contamos con dos

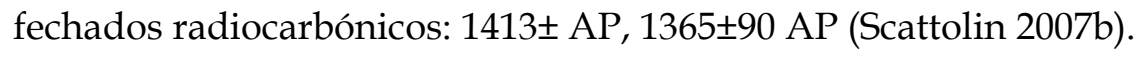

Los tiestos de la localidad de La Ciénaga (valle del Hualfín) fueron seleccionados de las recolecciones superficiales realizadas por Salvador Debenedetti en la década de 1920, actualmente forman parte del acervo del Museo Etnográfico "J. B. Ambrosetti" (Colecciones de las XIX, XX y XXIII expediciones de la Facultad de Filosofía y Letras - UBA). Por sus características estilísticas, estos fragmentos pueden ser asociados las producciones alfareras del período estudiado (Zagorodny y Balesta 2005).

El sitio Los Viscos es un alero de grandes dimensiones que se emplaza en el sector meridional del valle de El Bolsón (Departamento Belén). Este fue utilizado como refugio temporal ocupado de forma discontinua en un lapso que comprende ca. 1000 A.C - 1440 D.C. y estuvo relacionado a prácticas de interacción macrorregional, entre las que se menciona el tráfico caravanero (Korstanje y Würschmidt 1999; Korstanje 2005, 2007; Ávila y Puente 2008; Puente 2017). Las muestras incorporadas a este trabajo proceden de recolecciones superficiales y excavaciones sistemáticas realizadas por Korstanje y equipo en los años 1997 y 2007. Estos materiales se ubican cronológicamente dentro del período

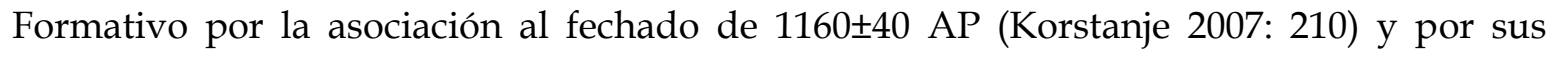
características estilísticas.

\section{La muestra y las categorías estilísticas utilizadas}

La muestra total analizada es de 78 cortes delgados, que corresponden a vasijas diferentes (Tabla 2). La mayor cantidad de fragmentos estudiados provienen de los sitios del sur Calchaquí y corresponden a 15 tiestos de grandes piezas clasificadas como Ordinario Alisado, Ordinario Pulido, Intermedio Alisado e Intermedio Baño Blanco, y 41 cortes realizados sobre ejemplares de la vajilla de servicio de distintos estilos: Gris Pulido, Gris Pulido Inciso, Gris Pulido Grabado, Ante Pulido, Ante Pulido Inciso, Ante Pulido Pintado; Candelaria Acanalado, Aguada Gris Grabado, San Rafael Grabado, Guachipas Polícromo, Marrón sobre Ante, Aguada Pintado, San Rafael Grabado (Figuras 2 y 3).

Para el valle de Hualfín contamos con las 10 muestras que se corresponden a fragmentos que han sido clasificados como Aguada Hualfín Gris Grabado, Aguada Pintado y Ciénaga III (Figura 4). 


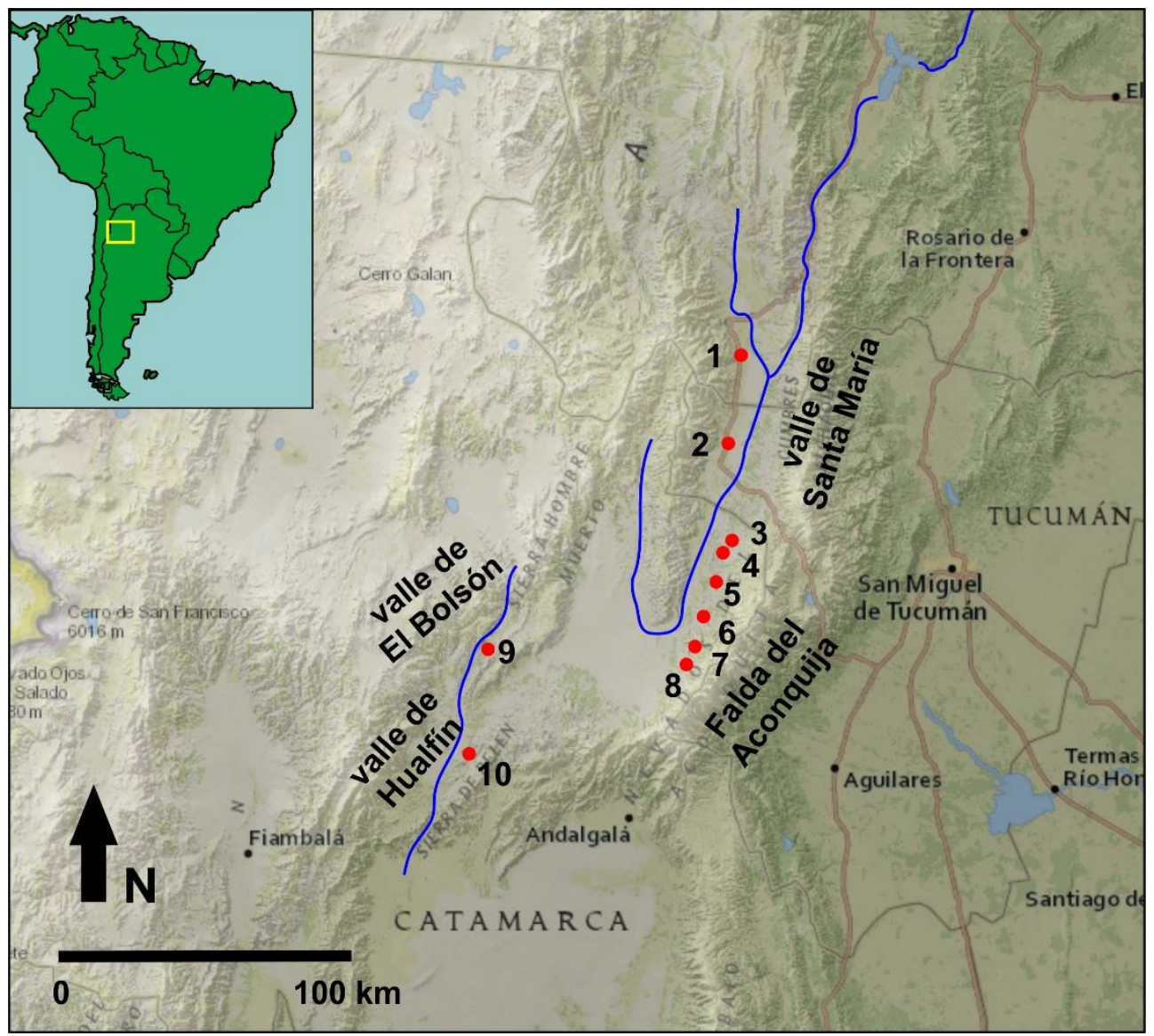

Figura 1. Área de estudio y sitios de procedencia de las muestras estudiadas. 1. Tolombón; 2. Bañado Viejo; 3. Caspinchango-Mesa Redonda; 4. Masao; 5. Ampajango; 6. Morro de las Espinillas; 7. Tesoro I; 8. Loma Alta; 9. Los Viscos; 10. La Ciénaga.

\begin{tabular}{|c|c|c|c|c|c|c|}
\hline Procedencia & Código & Fechado AP & Cal 1 o & Cal 2o & material & Referencia \\
\hline Los Viscos & UGA \#8362 & $1.160 \pm 40$ & $\begin{array}{l}780 a \\
900\end{array}$ & $\begin{array}{l}680 \mathrm{a} \\
900\end{array}$ & $\begin{array}{l}\text { marlo de } \\
\text { maíz }\end{array}$ & $\begin{array}{l}\text { Korstanje } \\
2005,2007\end{array}$ \\
\hline $\begin{array}{l}\text { Loma Alta } \\
\text { R19/3 }\end{array}$ & AA60336 & $1.413 \pm 32$ & $\begin{array}{l}617 a \\
660\end{array}$ & $\begin{array}{l}560 a \\
680\end{array}$ & $\begin{array}{l}\text { carbón } \\
\text { vegetal }\end{array}$ & $\begin{array}{l}\text { Scattolin } \\
2007 \mathrm{~b}\end{array}$ \\
\hline Loma Alta R88/4/3 & GX21577 & $1.365 \pm 90$ & $\begin{array}{l}600 a \\
780\end{array}$ & $\begin{array}{l}530 \mathrm{a} \\
900\end{array}$ & $\begin{array}{l}\text { carbón } \\
\text { vegetal }\end{array}$ & $\begin{array}{l}\text { Scattolin } \\
2007 \mathrm{~b}\end{array}$ \\
\hline $\begin{array}{l}\text { Tesoro I } \\
\text { NA C2 N } 10\end{array}$ & AA60337 & $1.251 \pm 31$ & $\begin{array}{l}680 a \\
810\end{array}$ & $\begin{array}{l}680 a \\
890\end{array}$ & $\begin{array}{l}\text { carbón } \\
\text { vegetal }\end{array}$ & $\begin{array}{l}\text { Scattolin } \\
2007 \mathrm{~b}\end{array}$ \\
\hline $\begin{array}{l}\text { Tesoro I } \\
\text { S1 N4 }\end{array}$ & GX21582-G & $1.090 \pm 115$ & $\begin{array}{l}770 \mathrm{a} \\
1040\end{array}$ & $\begin{array}{l}680 \mathrm{a} \\
1190\end{array}$ & $\begin{array}{l}\text { carbón } \\
\text { vegetal }\end{array}$ & $\begin{array}{l}\text { Scattolin } \\
2007 \mathrm{~b}\end{array}$ \\
\hline $\begin{array}{l}\text { Morro de las Espinillas } \\
\text { S5 /N5 }\end{array}$ & AA 32430 & $1.160 \pm 40$ & $\begin{array}{l}780 \mathrm{a} \\
960\end{array}$ & $\begin{array}{l}770 \mathrm{a} \\
980\end{array}$ & $\begin{array}{l}\text { carbón } \\
\text { vegetal }\end{array}$ & $\begin{array}{l}\text { Scattolin } \\
2007 \mathrm{~b}\end{array}$ \\
\hline $\begin{array}{l}\text { Morro de las Espinillas } \\
\text { S3 /N5 }\end{array}$ & GX 25168 & $1.140 \pm 70$ & $\begin{array}{l}780 \mathrm{a} \\
990\end{array}$ & $\begin{array}{l}710 \mathrm{a} \\
1.030 \\
\end{array}$ & $\begin{array}{l}\text { carbón } \\
\text { vegetal }\end{array}$ & $\begin{array}{l}\text { Scattolin } \\
2007 \mathrm{~b}\end{array}$ \\
\hline $\begin{array}{l}\text { Bañado Viejo } \\
\text { S1 / N4 }\end{array}$ & LP 923 & $1.170 \pm 40$ & $\begin{array}{l}780 a \\
940\end{array}$ & $\begin{array}{l}770 \mathrm{a} \\
980\end{array}$ & $\begin{array}{l}\text { carbón } \\
\text { vegetal }\end{array}$ & $\begin{array}{l}\text { Scattolin } \\
2007 \mathrm{~b}\end{array}$ \\
\hline
\end{tabular}

Tabla 1. Fechados radiocarbónicos de los sitios bajo estudio. 


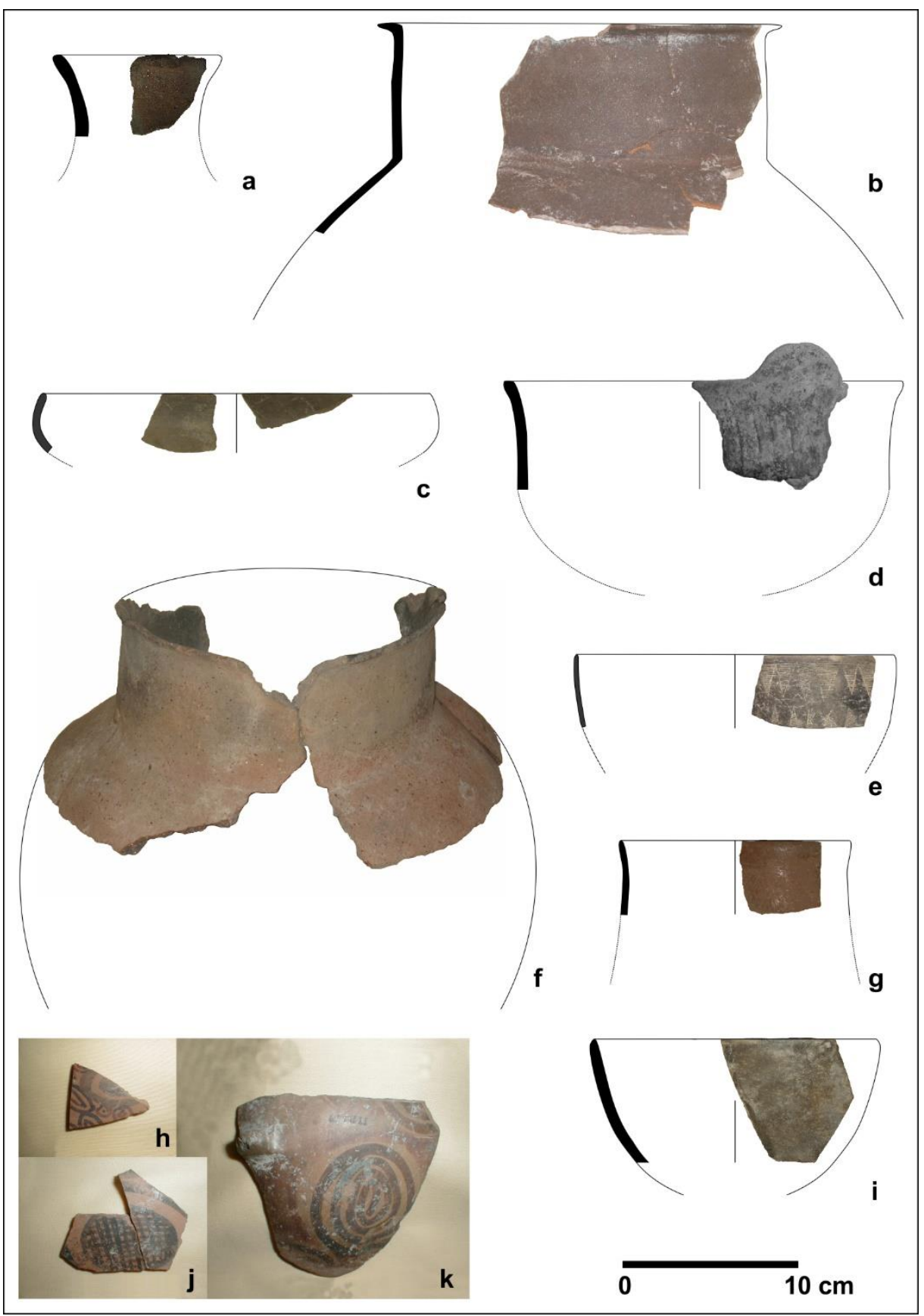

Figura 2. Ejemplos alfareros analizados procedentes del sur Calchaquí. a. Cántaro Ordinario Alisado 369-10 (Loma Alta); b. Tinaja Marrón sobre Ante 448-15 (Morro de las Espinillas); c. Escudilla Gris Pulido Inciso 389-6 (Loma Alta); d. Cuenco Candelaria Acanalado 448-17 (Morro de las Espinillas); e. Cuenco Gris Pulido Grabado 560-T3 (Tesoro I); f. Tinaja Ordinario Alisado 443-2 (Morro de las Espinillas); g. Jarra Ante Pulido Inciso 378-11 (Loma Alta); h. Fragmento estilo Guachipas Polícromo Corte 9 (Ampajango); i. Cuenco Gris Pulido 403-1 (Bañado Viejo), j. Fragmento estilo Aguada Pintado Laz113 (Ampajango); k. Fragmento estilo Guachipas Polícromo Laz112 (Ampajango). 


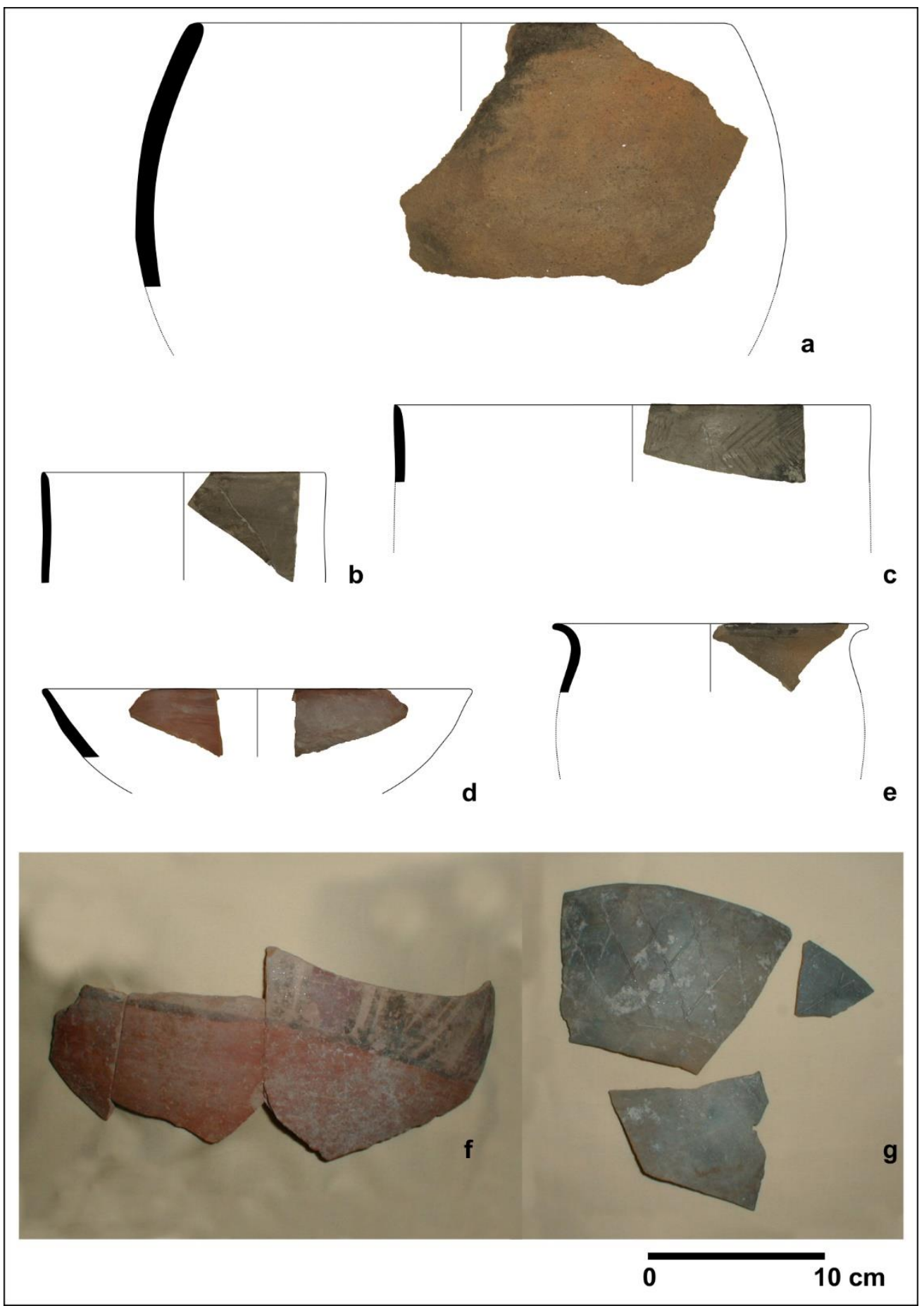

Figura 3. Ejemplos alfareros analizados procedentes del sur Calchaquí. a. Olla Intermedio Baño Blanco 552-T1 (Tesoro I); b. Jarro Gris Pulido 401-B4-25 (Bañado Viejo); c. Jarro Gris Pulido Inciso 365-25 (Loma Alta); d. Escudilla Ante Pulido Pintado 402-6 (Bañado Viejo); e. Olla Intermedio Alisado 587-T1 (Tesoro I); f. Fragmento de vasija de cuerpo globular de estilo Guachipas Polícromo Corte 13 (Masao); g. Cuenco San Rafael Gris Grabado 454-1 (Morro de las Espinillas). 


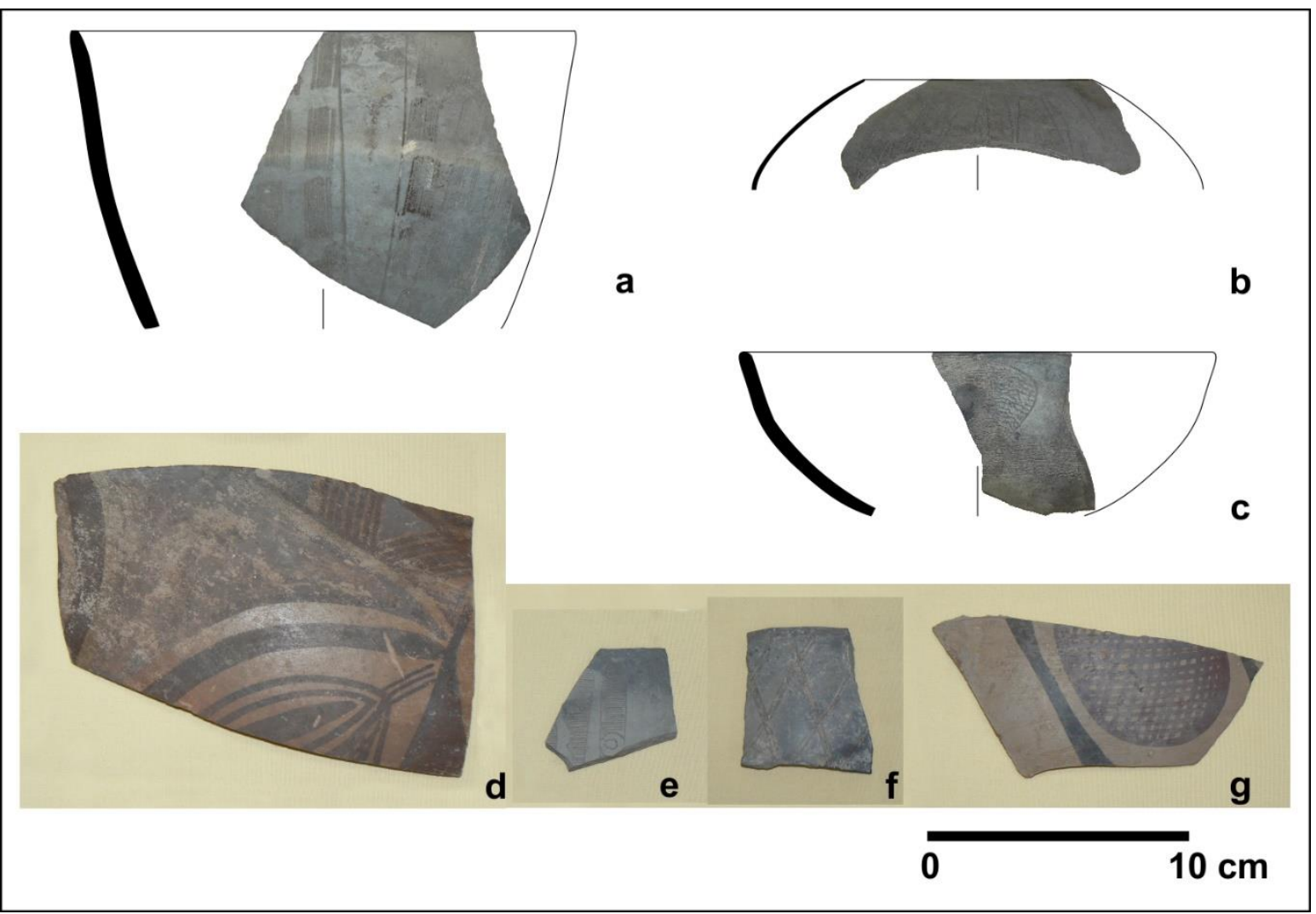

Figura 4. Ejemplos alfareros analizados procedentes del sitio La Ciénaga. Colecciones correspondientes a las XX y XXIII expediciones de la Facultad de Filosofía y Letras, Museo Etnográfico. a. Cuenco profundo Aguada Hualfín Gris Grabado (32108); b. Ollita Ciénaga III (32214); c. Cuenco Aguada Hualfín Gris Grabado (32083); d. Fragmento Aguada Pintado (33899);

e. Fragmento Aguada Hualfín Gris Grabado (31907); f. Fragmento Aguada Hualfín Gris Grabado (32185); g. Fragmento Aguada Pintado (31981).

Con respecto al valle de El Bolsón, se analizaron 2 muestras de cerámica ordinaria y 10 de estilo Aguada Hualfín Gris Grabado y Aguada Pintado (Figura 5), según las clasificaciones propuestas por González (1977).

A través de la historia de los estudios cerámicos en el Noroeste argentino, se crearon categorías para referenciar las alfarerías de cada una de las regiones investigadas, aunque compartieran el rango temporal y semejanzas estilísticas. Esta invención de múltiples nombres -i.e. Guachipas Polícromo, Aguada Pintado, Rupachico Polícromo, Aguada Hualfín Gris Grabado, Candelaria Gris Grabado, etc.- correspondió a la necesidad de dar cuenta de las particularidades de los materiales en relación con sus lugares de hallazgo. Sin embargo, este criterio de clasificación enmascaró la posibilidad de distinguir la procedencia local o alóctona de dichos bienes y generó, en muchos casos, confusión al estudiarlos desde una escala regional más amplia (Scattolin 2006b, 2007a). 


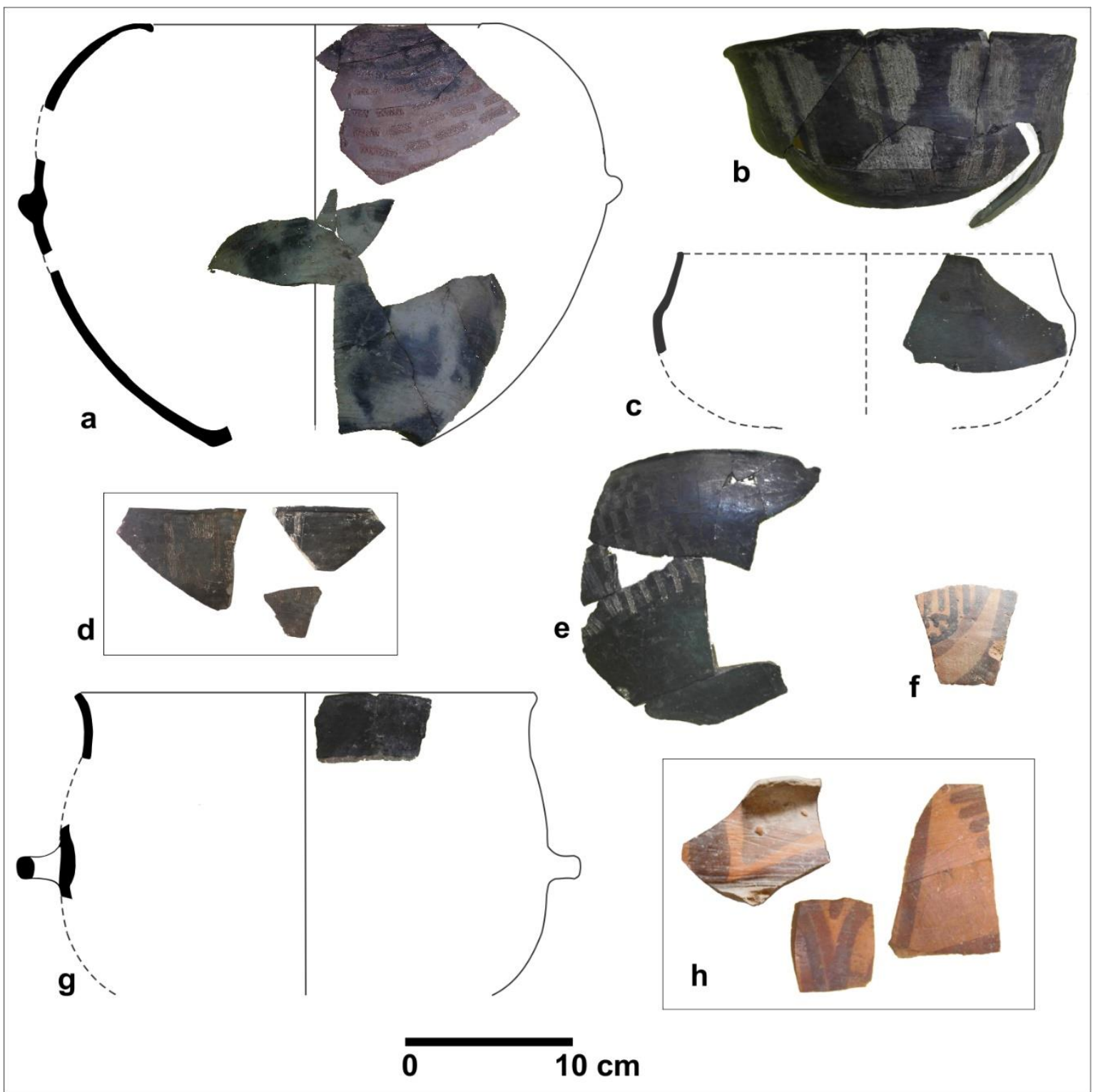

Figura 5. Ejemplos alfareros analizados procedentes del sitio Los Viscos. a. Cántaro Aguada Hualfín Gris Grabado (v37); b. puco Aguada Hualfín Gris Grabado (v313); c. puco Aguada Hualfín Gris Grabado (6(1)352); d. fragmentos de puco Aguada Hualfín Gris Grabado (r108); e. puco Aguada Hualfín Gris Grabado (v449); f. fragmento Aguada Pintado (r377); g. olla ordinaria (v131); h. fragmentos Aguada Pintado (v458).

Entendemos que estas alfarerías son el resultado de elecciones técnicas y estéticas compartidas que trascienden las áreas de estudio específicas donde fueron recuperadas. Es decir, conforman un conjunto cerámico con un 'aire de familia' -buena cochura, patrones de bicromía y policromía, uso de la técnica del grabado, superficies pulidas grises y/o negras, etc.- pero reconocemos las variaciones locales dadas por el despliegue iconográfico 
diferencial de diseños plásticos policromos no figurativos y geométricos, triángulos curvos, volutas y punteado, cabezas trofeos, felinos, el 'sacrificador' y/o el escobado inciso-grabado formando dameros o haces de líneas (ver Scattolin 2007a para un desarrollo completo de la problemática).

De este modo, debido a la diversidad de nomenclaturas que se han utilizado para reconocer los tipos cerámicos presentes en las regiones estudiadas, decidimos diferenciar estilísticamente la muestra según categorías más inclusivas, pensadas a partir de atributos estéticos similares. La clasificación estilística usada en este trabajo contempla los conceptos de:

-Aguada Pintado, reúne los fragmentos que han sido clasificados también como Guachipas Polícromo, San Rafael Pintado, Aguada Bicolor, Aguada Decadente, Rupachico Polícromo, entre otros (ver Scattolin 2006b y 2007a para una discusión de las nomenclaturas asociadas). -Aguada Grabado, término que agrupa las piezas correspondientes al Aguada Hualfín Gris Grabado, San Rafael Grabado, Ciénaga III, Punta Colorada Grabado, Las Mercedes, Candelaria Gris Grabado y Candelaria Negro Grabado, entre otras denominaciones (ver Scattolin 2006b y 2007a para una discusión de las nomenclaturas asociadas).

-Pulido Fino, corresponde a piezas clasificadas como Gris Pulido, Gris Pulido Inciso, Ante Pulido, Ante Pulido Inciso, Ante Pulido Pintado, etc. (Scattolin 1990, 2006b, 2007a; Bugliani 2008; Pereyra Domingorena 2010; Puente 2017).

-Ordinario, agrupa el Ordinario Alisado, Ordinario Pulido, Intermedio Alisado.

-Baño Blanco (Scattolin 2007a; Bugliani 2008; Pereyra Domingorena 2010).

-Candelaria Acanalado (Scattolin 2007a) (Tabla 2).

Por lo anteriormente expuesto, aquí utilizamos las unidades estilísticas tradicionales, como por ejemplo Aguada, en un sentido amplio, sin anclarnos a la idea de una "cultura de origen andino" (en el sentido de González 1964) centrada en un determinado espacio geográfico (valle de Hualfín o valle de Ambato) y su hipotética expansión territorial/cultural.

\section{Petrografía cerámica: variables y procedimientos analíticos}

El análisis petrográfico se realizó con microscopio óptico de luz transmitida. Las láminas delgadas se confeccionaron respetando el eje vertical de la pieza y tienen un espesor de 0,03 mm. El estudio consistió en la clasificación tanto de las inclusiones no plásticas como de los poros y/o cavidades y la identificación de las estructuras de los fondos de pasta. El análisis realizado es cualitativo y cuantitativo, es decir, se registran los estados de atributos 
de las variables mencionadas $\mathrm{y}$, además se cuantifica la distribución modal de los componentes.

Esta técnica permitió identificar clastos minerales, fragmentos de roca, vidrio vesicular $(\mathrm{Vv})$, gránulos de arcilla, minerales opacos $(\mathrm{Op})$ y tiesto molido (TM) o chamote. Los clastos minerales corresponden a fragmentos de cristales tales como cuarzo (Qz), feldespato potásico (Fk), plagioclasas (Pl), microclino (Mr), anfíboles-piroxenos (Af-Px), biotita (Bi) y muscovita $(\mathrm{Mu})$, granate y turmalina. Los fragmentos de roca se caracterizan de modo general en volcánicos (LV), graníticos (LG), metamórficos (LM) y sedimentarios (LS), según sea su origen.

Además de la identificación de su naturaleza, se reconoció el grado de esfericidad (alta y baja) y redondez (de muy anguloso a muy redondeado) del temperante, utilizándose el gráfico de estimación visual de Barraclough publicado en Orton y colaboradores (1997: 268). Finalmente, se registró el tamaño de los elementos no plásticos siguiendo la clasificación granulométrica propuesta por Wentworth (Adams et al. 1997 [1984]). En las muestras analizadas los tamaños reconocidos son: limo grueso $(0,03-0,06 \mathrm{~mm})$, arena muy fina $(0,06-$ $0,125 \mathrm{~mm})$, arena fina $(0,125-0,25 \mathrm{~mm})$, arena mediana $(0,25-0,5 \mathrm{~mm})$, arena gruesa $(0,5-1$ $\mathrm{mm})$, arena muy gruesa $(1-2 \mathrm{~mm})$ y canto pequeño $(2-4 \mathrm{~mm})$.

Dentro de la categoría fondo de pasta o matriz se observan dos variables: el color y la estructura. El primero de ellos se registra sin polarizador, se lo denomina de forma genérica indicando además si hay gradación en la tonalidad. En tanto que la estructura de fondo refiere a la matriz arcillosa cocida y a las inclusiones de tamaño inferior a 0,015 mm (Courtois 1976). Su clasificación se ve afectada por las texturas originales de las gredas utilizadas y por las temperaturas alcanzadas durante la cocción de las vasijas (Cremonte 1996). Para este análisis se utilizaron las siguientes categorías modificadas a partir de la propuesta de Courtois (1976):

- pseudolepidoblástica: masa de laminillas de micas detríticas abundantes y muy pequeñas unidas por una escasa fracción de arcilla.

- criptofilitosa: matriz arcillosa muy cementada en la que no es posible identificar las microlaminillas de micas.

- microgranosa: abundantes cantidades de microcristales no arcillosos que por su tamaño pequeño no es posible identificarlos con exactitud.

Para la clasificación de las cavidades y/o poros se tuvieron en cuenta la forma general (alargada, redondeada, irregular) y el tamaño medido en $\mathrm{mm}$. 
El último paso de este análisis se corresponde con el cálculo de los porcentajes de los componentes que integran la pasta (inclusiones, matriz y cavidades). Para ello, se efectuó un conteo mínimo de 300 puntos (point counting) por corte, utilizando el método de intercepción múltiple (multiple interseption) (Ownby 2010).

La cuantificación obtenida fue sometida al análisis estadístico para estudiar las relaciones de similitud y diferenciación existentes entre las muestras en relación con sus componentes. Para ello se aplicó el Análisis de Conglomerados y el Análisis de Componentes Principales (ACP), ambos ensayos fueron realizados con el programa PAST versión 2.17 (Hammer et al. 2001). El primero de ellos es una técnica exploratoria y el segundo, multivariada. Ambas constituyen análisis estadísticos complementarios para determinar un ordenamiento de los objetos considerando múltiples características (Shennan 1992; Bugliani 2007).

A partir de las relaciones establecidas entre el porcentaje de matriz, la densidad de inclusiones, su granulometría y las estructuras de fondo de la matriz arcillosa se propusieron modalidades de elaboración de las pastas cerámicas.

\section{Modalidades de pastas cerámicas}

Los análisis cuantitativos y cualitativos permitieron determinar nueve modalidades de manufacturas alfareras, las cuales se clasificaron como pastas finas, medias y gruesas.

Pastas finas

Se identificaron tres modalidades de pastas finas (Figura 6):

M1. Modalidad sin agregado de inclusiones. El material no plástico registrado se corresponde a clastos naturales de la greda.

M2. Modalidad con agregado de clastos cuya granulometría varía entre limo grueso y arena muy fina (0,03 - 0,125 mm), que representan más del $9 \%$ de la pasta.

M3. Modalidad con agregado de inclusiones cuyo granulometría oscila entre limo grueso y arena fina $(0,03-0,25 \mathrm{~mm})$, cuya densidad varía entre el $12 \%$ y el $40 \%$. 

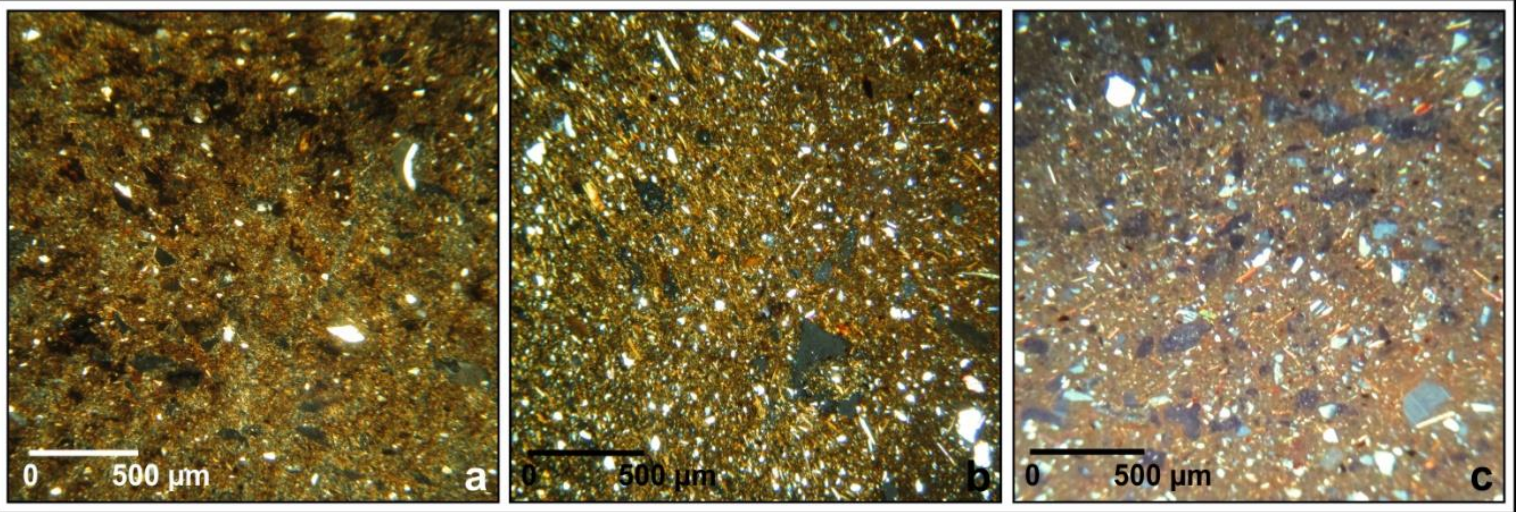

Figura 6. Fotomicrografías de las pastas finas. a. M1 (370-517); b. M2 (Corte 6); c. M3 (r377).

Pastas medias

Se identificaron cuatro pastas medias (Figura 7):

M4. Modalidad con temperante de tamaño seleccionado con una granulometría regular que oscila entre arena media y arena gruesa $(0,25 \mathrm{~mm}$ a $1 \mathrm{~mm})$. El porcentaje de inclusiones se aproxima al $16 \%$.

M5. Modalidad con temperante de tamaño seleccionado con una granulometría regular que oscila entre arena gruesa y arena muy gruesa $(0,50 \mathrm{~mm}$ a $2 \mathrm{~mm})$. El porcentaje de inclusiones oscila entre el $21 \%$ y $31 \%$ de la pasta. Esta modalidad también se caracteriza por presentar estructuras de fondo criptofilitosas.

M6. Modalidad con el agregado de inclusiones no plásticas de tamaño no seleccionado cuya granulometría oscila entre limo grueso y arena muy gruesa $(0,03 \mathrm{~mm}$ a $2 \mathrm{~mm})$. La densidad del temperante ronda el 30\%.

M7. Modalidad con temperantes tamaño no seleccionado (amplio rango granulométrico) que se diferencia de la anterior dado que las inclusiones varían entre limo grueso y arena gruesa $(0,03$ a $1 \mathrm{~mm})$. Se trata de un solo ejemplar cuya densidad de material no plástico es de $29 \%$.

Pastas gruesas

Se identificaron dos modalidades (Figura 8):

M8. Modalidad con agregado de inclusiones de tamaño no seleccionado cuya granulometría oscila entre limo medio y arena muy gruesa $(0,015 \mathrm{~mm}$ a $2 \mathrm{~mm})$, aunque predominan las arenas de tipo gruesa y muy gruesa $(0,5 \mathrm{~mm}$ a $2 \mathrm{~mm})$. La densidad del temperante es 
superior al 30\%. Además se caracteriza por presentar estructuras de fondo pseudolepidoblásticas.

M9. Modalidad con agregado de inclusiones de tamaños no seleccionado cuya granulometría oscila entre limo grueso y canto pequeño $(0,03 \mathrm{~mm}$ a $4 \mathrm{~mm})$, aunque predominan los de tamaño arena gruesa y arena muy gruesa $(0,5 \mu \mathrm{m}$ a $2 \mathrm{~mm})$. Se trata de un solo ejemplar cuya densidad de inclusiones es de $38 \%$ y es el único caso que posee temperante de tiesto molido $(8,32 \%$ del total de la pasta) junto a clastos volcánicos.

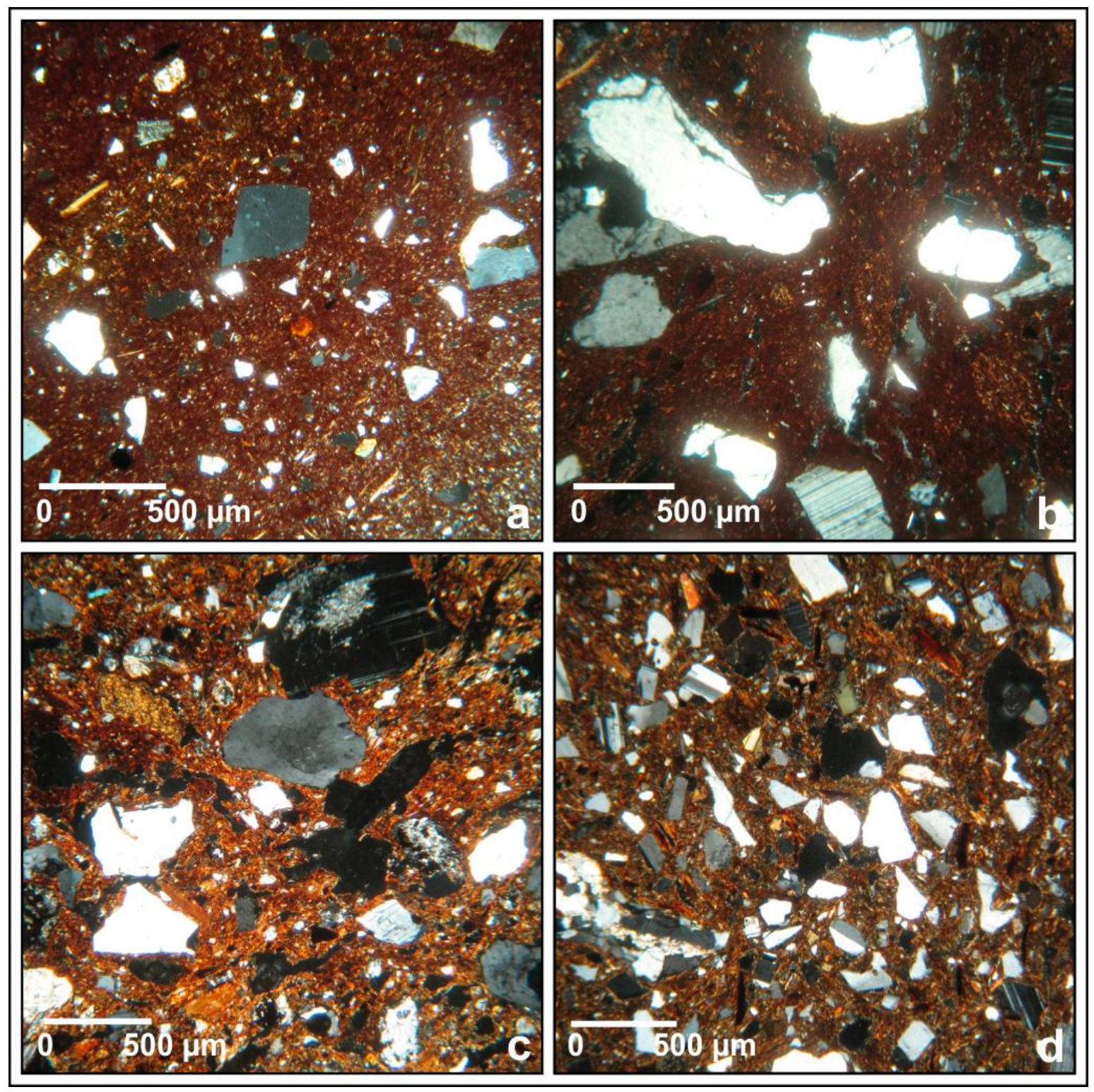

Figura 7. Fotomicrografías de pastas medias. a. M4 (Corte 7); b. M5 (454-6); c. M6 (401-M8); d. M7 (587-T1). 


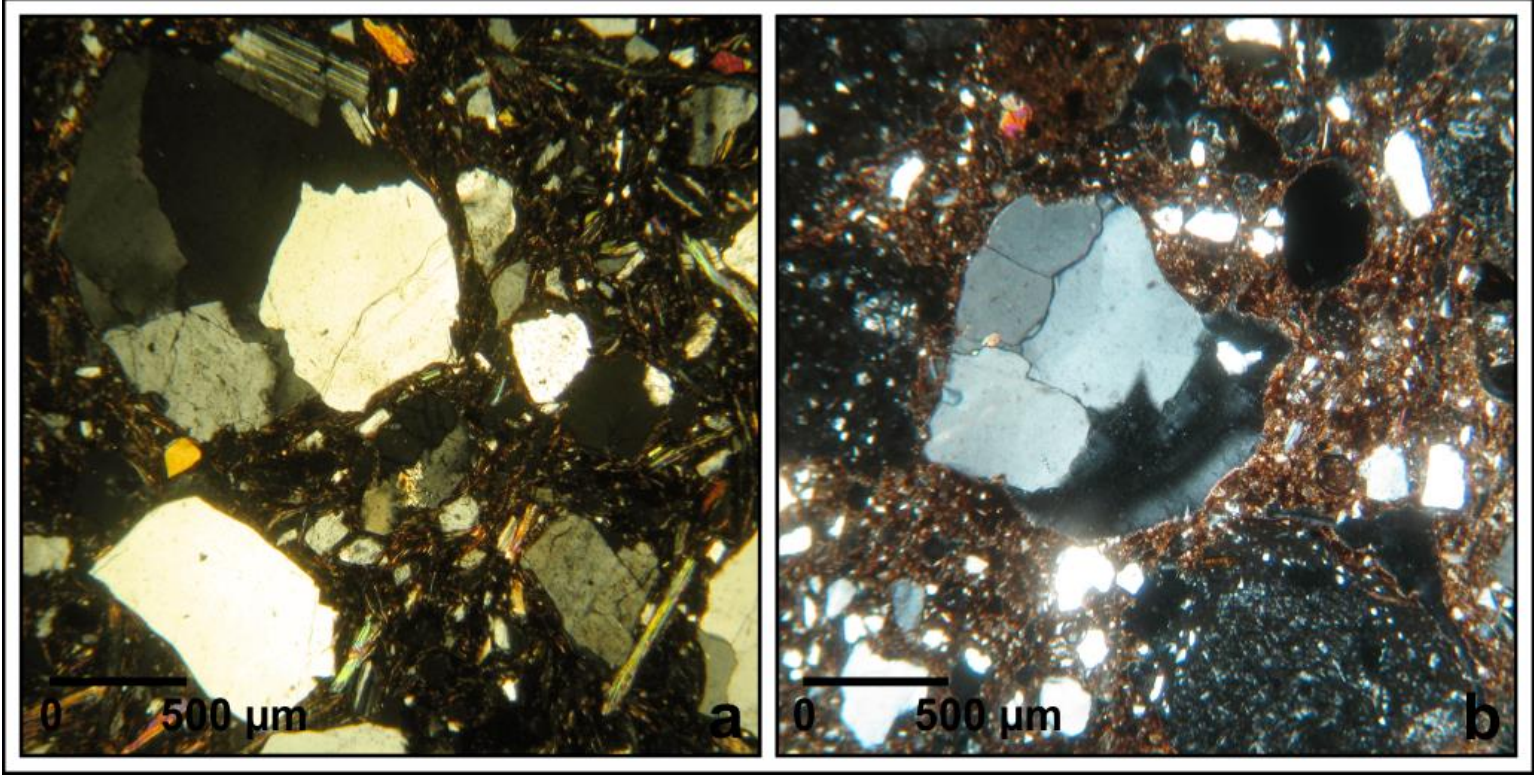

Figura 8. Fotomicrografías de pastas gruesas. a. M8 (370-253); b. M9 (405-6).

En la tabla 2 se sintetizan las vinculaciones entre los 78 los casos de estudio, los lugares de hallazgo y los estilos cerámicos asociados con los tipos de pasta cerámica y sus modalidades conformados a partir de nuestros análisis. Luego, con la intensión de determinar si dichas modalidades se vinculaban con aspectos composicionales, se procedió a analizar la relación de las muestras entre sí respecto a la naturaleza y proporción de sus componentes.

\begin{tabular}{|c|c|c|c|c|c|}
\hline Caso & Procedencia & $\begin{array}{c}\text { Denominación } \\
\text { específica }\end{array}$ & Estilo & Pasta & Modalidad \\
\hline Laz112 & Ampajango & $\begin{array}{l}\text { Guachipas } \\
\text { Polícromo }\end{array}$ & Aguada Pintado & \multirow{6}{*}{ Fina } & \multirow{6}{*}{1} \\
\hline Laz113 & Ampajango & $\begin{array}{l}\text { Aguada } \\
\text { Pintado }\end{array}$ & Aguada Pintado & & \\
\hline $447-20$ & $\begin{array}{l}\text { Morro de las } \\
\text { Espinillas }\end{array}$ & $\begin{array}{l}\text { Aguada } \\
\text { Pintado }\end{array}$ & Aguada Pintado & & \\
\hline $370-517$ & Loma Alta & $\begin{array}{l}\text { Aguada Gris } \\
\text { Grabado }\end{array}$ & $\begin{array}{l}\text { Aguada } \\
\text { Grabado }\end{array}$ & & \\
\hline $454-1$ & $\begin{array}{l}\text { Morro de las } \\
\text { Espinillas }\end{array}$ & $\begin{array}{l}\text { San Rafael } \\
\text { Grabado }\end{array}$ & $\begin{array}{l}\text { Aguada } \\
\text { Grabado }\end{array}$ & & \\
\hline $447-7$ & $\begin{array}{l}\text { Morro de las } \\
\text { Espinillas }\end{array}$ & $\begin{array}{l}\text { San Rafael } \\
\text { Grabado }\end{array}$ & $\begin{array}{l}\text { Aguada } \\
\text { Grabado }\end{array}$ & & \\
\hline
\end{tabular}




\begin{tabular}{|c|c|c|c|c|c|}
\hline Caso & Procedencia & $\begin{array}{c}\text { Denominación } \\
\text { específica }\end{array}$ & Estilo & Pasta & Modalidad \\
\hline 31907 & La Ciénaga & $\begin{array}{l}\text { Aguada } \\
\text { Hualfín Gris } \\
\text { Grabado }\end{array}$ & $\begin{array}{l}\text { Aguada } \\
\text { Grabado }\end{array}$ & \multirow{7}{*}{ Fina } & \multirow{7}{*}{1} \\
\hline 32192 & La Ciénaga & $\begin{array}{l}\text { Aguada } \\
\text { Hualfín Gris } \\
\text { Grabado }\end{array}$ & $\begin{array}{l}\text { Aguada } \\
\text { Grabado }\end{array}$ & & \\
\hline 32083 & La Ciénaga & $\begin{array}{l}\text { Aguada } \\
\text { Hualfín Gris } \\
\text { Grabado }\end{array}$ & $\begin{array}{l}\text { Aguada } \\
\text { Grabado }\end{array}$ & & \\
\hline $454-4$ & $\begin{array}{l}\text { Morro de las } \\
\text { Espinillas }\end{array}$ & $\begin{array}{l}\text { San Rafael } \\
\text { Grabado }\end{array}$ & $\begin{array}{l}\text { Aguada } \\
\text { Grabado }\end{array}$ & & \\
\hline $380-13$ & Loma Alta & Gris Pulido & Pulido Fino & & \\
\hline $378-7$ & Loma Alta & Gris Pulido & Pulido Fino & & \\
\hline $378-12$ & Loma Alta & Gris Pulido & Pulido Fino & & \\
\hline V297 & Los Viscos & $\begin{array}{l}\text { Aguada } \\
\text { Pintado }\end{array}$ & Aguada Pintado & \multirow{10}{*}{ Fina } & \multirow{10}{*}{2} \\
\hline V458 & Los Viscos & $\begin{array}{l}\text { Aguada } \\
\text { Pintado }\end{array}$ & Aguada Pintado & & \\
\hline Corte 9 & Ampajango & $\begin{array}{l}\text { Guachipas } \\
\text { Polícromo }\end{array}$ & Aguada Pintado & & \\
\hline Corte 6 & Tolombón & $\begin{array}{l}\text { Aguada } \\
\text { Pintado }\end{array}$ & Aguada Pintado & & \\
\hline 31981 & La Ciénaga & $\begin{array}{l}\text { Aguada } \\
\text { Pintado }\end{array}$ & Aguada Pintado & & \\
\hline 33899 & La Ciénaga & $\begin{array}{l}\text { Aguada } \\
\text { Pintado }\end{array}$ & Aguada Pintado & & \\
\hline 31995 & La Ciénaga & $\begin{array}{l}\text { Aguada } \\
\text { Pintado }\end{array}$ & Aguada Pintado & & \\
\hline Corte 12 & Caspinchango & $\begin{array}{l}\text { Aguada } \\
\text { Pintado }\end{array}$ & Aguada Pintado & & \\
\hline $448-18$ & $\begin{array}{l}\text { Morro de las } \\
\text { Espinillas }\end{array}$ & $\begin{array}{l}\text { Aguada } \\
\text { Pintado }\end{array}$ & Aguada Pintado & & \\
\hline v37 & Los Viscos & $\begin{array}{l}\text { Aguada } \\
\text { Hualfín Gris } \\
\text { Grabado }\end{array}$ & $\begin{array}{l}\text { Aguada } \\
\text { Grabado }\end{array}$ & & \\
\hline
\end{tabular}




\begin{tabular}{|c|c|c|c|c|c|}
\hline Caso & Procedencia & $\begin{array}{c}\text { Denominación } \\
\text { específica }\end{array}$ & Estilo & Pasta & Modalidad \\
\hline 32108 & La Ciénaga & $\begin{array}{l}\text { Aguada } \\
\text { Hualfín Gris } \\
\text { Grabado }\end{array}$ & $\begin{array}{l}\text { Aguada } \\
\text { Grabado }\end{array}$ & \multirow{19}{*}{ Fina } & \multirow{19}{*}{2} \\
\hline V313 & Los Viscos & $\begin{array}{l}\text { Aguada } \\
\text { Hualfín Gris } \\
\text { Grabado }\end{array}$ & $\begin{array}{l}\text { Aguada } \\
\text { Grabado }\end{array}$ & & \\
\hline R108 & Los Viscos & $\begin{array}{l}\text { Aguada } \\
\text { Hualfín Gris } \\
\text { Grabado }\end{array}$ & $\begin{array}{l}\text { Aguada } \\
\text { Grabado }\end{array}$ & & \\
\hline $365-25$ & Loma Alta & $\begin{array}{l}\text { Gris Pulido } \\
\text { Inciso }\end{array}$ & Pulido Fino & & \\
\hline $448-14$ & $\begin{array}{l}\text { Morro de las } \\
\text { Espinillas }\end{array}$ & $\begin{array}{l}\text { Gris Pulido } \\
\text { Inciso }\end{array}$ & Pulido Fino & & \\
\hline 560-T3 & Tesoro I & $\begin{array}{l}\text { Gris Pulido } \\
\text { Grabado }\end{array}$ & $\begin{array}{l}\text { Aguada } \\
\text { Grabado }\end{array}$ & & \\
\hline 32185 & La Ciénaga & $\begin{array}{l}\text { Aguada Gris } \\
\text { Grabado }\end{array}$ & $\begin{array}{l}\text { Aguada } \\
\text { Grabado }\end{array}$ & & \\
\hline 32214 & La Ciénaga & Ciénaga III & $\begin{array}{l}\text { Aguada } \\
\text { Grabado }\end{array}$ & & \\
\hline 6 (1) 352 & Los Viscos & Gris Pulido & Pulido Fino & & \\
\hline $6(1) 473$ & Los Viscos & Gris Pulido & Pulido Fino & & \\
\hline $584-4$ & Loma Alta & Gris Pulido & Pulido Fino & & \\
\hline $378-28$ & Loma Alta & Gris Pulido & Pulido Fino & & \\
\hline $367-1$ & Loma Alta & Ante Pulido & Pulido Fino & & \\
\hline $402-12$ & Bañado Viejo & Gris Pulido & Pulido Fino & & \\
\hline $403-1$ & Bañado Viejo & Gris Pulido & Pulido Fino & & \\
\hline 401-B4-25 & Bañado Viejo & Gris Pulido & Pulido Fino & & \\
\hline $407-2$ & Bañado Viejo & Gris Pulido & Pulido Fino & & \\
\hline $402-6$ & Bañado Viejo & $\begin{array}{l}\text { Ante Pulido } \\
\text { Pintado }\end{array}$ & Pulido Fino & & \\
\hline $402-9$ & Bañado Viejo & $\begin{array}{l}\text { Ante Pulido } \\
\text { Pintado }\end{array}$ & Pulido Fino & & \\
\hline
\end{tabular}




\begin{tabular}{|c|c|c|c|c|c|}
\hline Caso & Procedencia & $\begin{array}{c}\text { Denominación } \\
\text { específica }\end{array}$ & Estilo & Pasta & Modalidad \\
\hline $448-15$ & $\begin{array}{l}\text { Morro de las } \\
\text { Espinillas }\end{array}$ & $\begin{array}{l}\text { Marrón sobre } \\
\text { Ante }\end{array}$ & Aguada Pintado & Fina & 2 \\
\hline $442-3$ & $\begin{array}{l}\text { Morro de las } \\
\text { Espinillas }\end{array}$ & $\begin{array}{l}\text { San Rafael } \\
\text { Grabado }\end{array}$ & $\begin{array}{l}\text { Aguada } \\
\text { Grabado }\end{array}$ & \multirow{7}{*}{ Fina } & \multirow{7}{*}{3} \\
\hline Corte 13 & Masao & $\begin{array}{l}\text { Guachipas } \\
\text { Polícromo }\end{array}$ & Aguada Pintado & & \\
\hline r377 & Los Viscos & $\begin{array}{l}\text { Aguada } \\
\text { Pintado }\end{array}$ & Aguada Pintado & & \\
\hline 29375 & La Ciénaga & $\begin{array}{l}\text { Aguada } \\
\text { Hualfín Gris } \\
\text { Grabado }\end{array}$ & $\begin{array}{l}\text { Aguada } \\
\text { Grabado }\end{array}$ & & \\
\hline v449 & Los Viscos & $\begin{array}{l}\text { Aguada } \\
\text { Hualfín Gris } \\
\text { Grabado }\end{array}$ & $\begin{array}{l}\text { Aguada } \\
\text { Grabado }\end{array}$ & & \\
\hline v312 & Los Viscos & $\begin{array}{l}\text { Aguada } \\
\text { Hualfín Gris } \\
\text { Grabado }\end{array}$ & $\begin{array}{l}\text { Aguada } \\
\text { Grabado }\end{array}$ & & \\
\hline $370-446$ & Loma Alta & $\begin{array}{l}\text { Gris Pulido } \\
\text { Inciso }\end{array}$ & Pulido Fino & & \\
\hline Corte 7 & Tolombón & $\begin{array}{l}\text { Guachipas } \\
\text { Polícromo }\end{array}$ & Aguada Pintado & \multirow{7}{*}{ Media } & \multirow{7}{*}{4} \\
\hline $370-390$ & Loma Alta & $\begin{array}{l}\text { Aguada } \\
\text { Pintado }\end{array}$ & Aguada Pintado & & \\
\hline $384-2$ & Loma Alta & $\begin{array}{l}\text { Gris Pulido } \\
\text { Inciso }\end{array}$ & Pulido Fino & & \\
\hline $368-35$ & Loma Alta & $\begin{array}{l}\text { Gris Pulido } \\
\text { Inciso }\end{array}$ & Pulido Fino & & \\
\hline $553-1$ & Tesoro I & Gris Pulido & Pulido Fino & & \\
\hline $566-1$ & Tesoro I & Gris Pulido & Pulido Fino & & \\
\hline $378-11$ & Loma Alta & $\begin{array}{l}\text { Ante Pulido } \\
\text { Inciso }\end{array}$ & Pulido Fino & & \\
\hline $442-1$ & $\begin{array}{l}\text { Morro de las } \\
\text { Espinillas }\end{array}$ & $\begin{array}{l}\text { Intermedio } \\
\text { Alisado }\end{array}$ & Ordinario & \multirow{2}{*}{ Media } & \multirow[t]{2}{*}{5} \\
\hline v131 & Los Viscos & Ordinario & Ordinario & & \\
\hline
\end{tabular}




\begin{tabular}{|c|c|c|c|c|c|}
\hline Caso & Procedencia & $\begin{array}{c}\text { Denominación } \\
\text { específica }\end{array}$ & Estilo & Pasta & Modalidad \\
\hline $449-16$ & $\begin{array}{l}\text { Morro de las } \\
\text { Espinillas }\end{array}$ & $\begin{array}{l}\text { Intermedio } \\
\text { Alisado }\end{array}$ & Ordinario & \multirow{5}{*}{ Media } & \multirow{5}{*}{5} \\
\hline $454-6$ & $\begin{array}{l}\text { Morro de las } \\
\text { Espinillas }\end{array}$ & $\begin{array}{l}\text { Intermedio } \\
\text { Alisado }\end{array}$ & Ordinario & & \\
\hline 552-T1 & Tesoro I & $\begin{array}{l}\text { Intermedio } \\
\text { Baño Blanco }\end{array}$ & Baño Blanco & & \\
\hline r257 & Los Viscos & Ordinario & Ordinario & & \\
\hline $448-17$ & $\begin{array}{l}\text { Morro de las } \\
\text { Espinillas }\end{array}$ & $\begin{array}{l}\text { Candelaria } \\
\text { Acanalado }\end{array}$ & $\begin{array}{l}\text { Candelaria } \\
\text { Acanalado }\end{array}$ & & \\
\hline 401-A7 & Bañado Viejo & $\begin{array}{l}\text { Intermedio } \\
\text { Baño Blanco }\end{array}$ & Baño Blanco & \multirow{3}{*}{ Media } & \multirow{3}{*}{6} \\
\hline 401-M8 & Bañado Viejo & $\begin{array}{l}\text { Intermedio } \\
\text { Alisado }\end{array}$ & Ordinario & & \\
\hline 402-R1 & Bañado Viejo & $\begin{array}{l}\text { Intermedio } \\
\text { Alisado }\end{array}$ & Ordinario & & \\
\hline 587-T1 & Tesoro I & $\begin{array}{l}\text { Intermedio } \\
\text { Alisado }\end{array}$ & Ordinario & Media & 7 \\
\hline $443-2$ & $\begin{array}{l}\text { Morro de las } \\
\text { Espinillas }\end{array}$ & $\begin{array}{l}\text { Ordinario } \\
\text { Alisado }\end{array}$ & Ordinario & \multirow{8}{*}{ Gruesa } & \multirow{8}{*}{8} \\
\hline $370-253$ & Loma Alta & $\begin{array}{l}\text { Ordinario } \\
\text { Alisado }\end{array}$ & Ordinario & & \\
\hline 378-M1 & Loma Alta & $\begin{array}{l}\text { Ordinario } \\
\text { Pulido }\end{array}$ & Ordinario & & \\
\hline $370-13$ & Loma Alta & $\begin{array}{l}\text { Ordinario } \\
\text { Alisado }\end{array}$ & Ordinario & & \\
\hline 587-T6 & Tesoro I & $\begin{array}{l}\text { Intermedio } \\
\text { Alisado }\end{array}$ & Ordinario & & \\
\hline $369-10$ & Loma Alta & $\begin{array}{l}\text { Ordinario } \\
\text { Alisado }\end{array}$ & Ordinario & & \\
\hline 407-R1 & Bañado Viejo & $\begin{array}{l}\text { Ordinario } \\
\text { Pulido }\end{array}$ & Ordinario & & \\
\hline $375-67$ & Loma Alta & $\begin{array}{l}\text { Ordinario } \\
\text { Alisado }\end{array}$ & Ordinario & & \\
\hline
\end{tabular}




\begin{tabular}{|r|l|l|l|l|c|}
\hline \multicolumn{1}{|l|}{ Caso } & Procedencia & $\begin{array}{l}\text { Denominación } \\
\text { específica }\end{array}$ & Estilo & Pasta & Modalidad \\
\hline $385-19$ & Loma Alta & $\begin{array}{l}\text { Ordinario } \\
\text { Alisado }\end{array}$ & Ordinario & Gruesa & 8 \\
\hline $405-4$ & Bañado Viejo & $\begin{array}{l}\text { Ordinario } \\
\text { Pulido }\end{array}$ & Ordinario & Gruesa & 9 \\
\hline
\end{tabular}

Tabla 2. Relación entre los casos, su procedencia y su asociación estilística alfarera con los tipos de pasta cerámica y sus modalidades.

\section{Caracterización composicional}

Con el propósito de determinar la existencia de grupos composicionales se compararon los resultados obtenidos de la cuantificación de las siguientes variables: matriz; cavidades; clastos minerales de cuarzo, feldespato potásico, plagioclasas, microclino, biotita, muscovita, turmalina, granate, anfíboles-piroxenos; litoclastos metamórficos, graníticos, volcánicos y sedimentarios; vidrio vesicular; tiesto molido y minerales opacos (Tabla 3).

Para ello en primer lugar, se aplicó el análisis de conglomerados utilizando el algoritmo de pares agrupados y la correlación como índice de distancia (Shennan 1992). Con este ensayo se obtuvo un coeficiente de correlación cofenética de 0,83 el cual indica que el fenograma resultante es una representación confiable de la matriz de distancia generada (Lanteri et al. 2005).

Se detectaron dos agrupamientos principales y tres casos aislados (Figura 9):

Conglomerado 1: está integrado por 60 muestras que se agrupan por la presencia de cuarzo y vidrio como clastos minerales principales. A su vez, se observan cuatro subdivisiones definidas a partir de la asociación estrecha a nivel composicional:

-Conglomerado 1-A: conformado por 45 muestras en las que predominan clastos de cuarzo $(2,04 \%-10,23 \%) \mathrm{y}$, en menor medida, vidrio vesicular $(\leq 6,5 \%)$ y plagioclasas $(0,10 \%-2,70 \%)$. -Conglomerado 1-B: integrado por cuatro muestras. En sus pastas predominan clastos de cuarzo $(9,56 \%-11,14 \%)$ junto a fragmentos de rocas graníticas, metamórficas, volcánicas y vidrio vesicular.

-Conglomerado 1-C: constituido por nueve muestras caracterizadas por el predominio de clastos de cuarzo $(9,13 \%-13,20 \%)$ y vidrio vesicular $(\leq 8,27 \%)$.

-Conglomerado 1-D: formado por dos muestras que se caracterizan por el predominio de vidrio vesicular $(9,33 \%$ - 10,57\%), plagioclasas $(3,66 \%-8,94 \%)$ y cuarzo $(3,66 \%-4,97 \%)$. 
Este cluster agrupa las muestras correspondientes a las distintas modalidades de pastas finas (M1, M2 y M3). Además, se detectaron ejemplares de modalidades medias (M4, M5 y M6).

Conglomerado 2: está integrado 15 ejemplares en los que predominan los clastos de cuarzo $(6,75 \%-20,68 \%)$ y granito $(1,79 \%-15,05 \%)$. Las muestras se diferencian entre sí en la relación porcentual entre estos dos componentes mayoritarios y en la combinación con otros minerales -biotita, muscovita, plagioclasas y feldespato potásico- característicos de las rocas graníticas. Algunas de estas muestras también poseen fragmentos de rocas metamórficas. Principalmente, los casos se asocian a la modalidad gruesa (M8) pero también hemos detectado algunos de pastas medias (M5, M6 y M7).

R377: esta muestra posee una alta densidad de las inclusiones (40\%), donde predominan clastos de cuarzo, vidrio vesicular, biotita, plagioclasas y feldespato potásico. Sin embargo, por la granulometría de los mismos $(0,03-0,25 \mathrm{~mm})$ fue integrada como una excepción dentro de la modalidad fina M3.

405-4: en esta muestra predominan los clastos de cuarzo, tiesto molido y litoclastos volcánicos, correspondiéndose a la modalidad M9.

407-R1: su pasta presenta principalmente clastos de cuarzo y fragmentos de rocas sedimentarias, metamórficas y graníticas, correspondiéndose a la modalidad M8.

\begin{tabular}{|c|c|c|c|c|c|c|c|c|c|c|c|c|c|}
\hline Muestra & $\mathbf{M z}$ & $\mathrm{Cv}$ & $\mathrm{Qz}$ & Fk & Pl & $\mathbf{B i}$ & $\mathbf{M u}$ & Af-Px & LM & LG & LS & LV & Vv \\
\hline 407-R1 & 50,18 & 9,44 & 12,41 & 0,92 & 0,37 & 0,55 & 0,37 & 0,00 & 9,26 & 5,51 & 10,45 & 0,00 & 0,00 \\
\hline $407-2$ & 84,36 & 5,21 & 6,16 & 0,00 & 0,47 & 0,95 & 0,47 & 0,10 & 0,00 & 0,00 & 0,00 & 0,47 & 1,71 \\
\hline $405-4$ & 54,90 & 7,13 & 18,02 & 0,79 & 0,99 & 0,19 & 0,19 & 0,19 & 0,19 & 0,00 & 0,00 & 6,34 & 1,19 \\
\hline $403-1$ & 81,91 & 4,02 & 8,19 & 0,14 & 0,86 & 2,59 & 0,29 & 0,14 & 0,00 & 0,43 & 0,00 & 0,00 & 1,29 \\
\hline 401-M8 & 65,68 & 7,28 & 10,76 & 1,11 & 0,47 & 1,26 & 0,16 & 0,32 & 3,16 & 4,43 & 0,00 & 2,05 & 1,90 \\
\hline 402-R1 & 61,76 & 5,39 & 15,26 & 3,05 & 0,72 & 1,61 & 0,72 & 0,18 & 3,95 & 1,79 & 0,00 & 0,18 & 1,26 \\
\hline $402-12$ & 84,47 & 5,43 & 6,18 & 0,00 & 0,45 & 1,21 & 0,00 & 0,45 & 0,00 & 0,00 & 0,00 & 0,15 & 1,51 \\
\hline $402-9$ & 80,60 & 5,74 & 7,92 & 0,00 & 0,27 & 1,64 & 0,27 & 0,00 & 0,27 & 0,00 & 0,00 & 0,56 & 2,46 \\
\hline $402-6$ & 72,42 & 4,83 & 9,13 & 1,03 & 0,52 & 2,41 & 0,69 & 0,86 & 1,38 & 0,00 & 0,00 & 2,24 & 4,31 \\
\hline 401-A7 & 66,72 & 6,63 & 11,14 & 2,41 & 0,60 & 1,51 & 0,30 & 0,30 & 2,71 & 2,11 & 0,00 & 1,51 & 3,16 \\
\hline 401-b4-25 & 82,37 & 4,82 & 5,94 & 0,39 & 0,56 & 2,23 & 0,18 & 0,18 & 0,00 & 0,37 & 0,00 & 0,18 & 2,60 \\
\hline $385-19$ & 62,03 & 5,77 & 14,42 & 0,48 & 0,96 & 3,61 & 1,20 & 0,48 & 0,00 & 8,89 & 0,00 & 0,00 & 0,96 \\
\hline $375-67$ & 54,95 & 9,70 & 17,76 & 2,96 & 1,81 & 0,00 & 2,96 & 0,00 & 0,00 & 8,39 & 0,98 & 0,00 & 0,00 \\
\hline $369-10$ & 58,14 & 7,60 & 20,68 & 0,78 & 1,31 & 2,35 & 1,57 & 0,26 & 2,35 & 5,50 & 0,00 & 0,00 & 0,52 \\
\hline $370-13$ & 62,50 & 5,68 & 15,68 & 0,45 & 1,14 & 2,50 & 2,50 & 0,00 & 0,00 & 6,82 & 0,00 & 0,23 & 0,00 \\
\hline 378-M1 & 56,37 & 7,16 & 17,09 & 2,31 & 0,92 & 1,85 & 1,38 & 0,23 & 0,00 & 11,08 & 0,23 & 0,00 & 0,00 \\
\hline $370-253$ & 55,90 & 6,65 & 14,85 & 0,66 & 2,44 & 0,00 & 4,21 & 0,44 & 0,00 & 14,19 & 0,44 & 0,00 & 0,00 \\
\hline $378-11$ & 81,89 & 2,78 & 8,17 & 1,47 & 0,49 & 0,32 & 0,32 & 0,00 & 0,00 & 2,78 & 1,14 & 0,16 & 0,00 \\
\hline $368-35$ & 85,97 & 0,61 & 7,32 & 2,44 & 0,61 & 0,61 & 0,61 & 0,00 & 0,00 & 0,61 & 0,00 & 0,00 & 0,00 \\
\hline $384-2$ & 81,73 & 3,32 & 8,07 & 0,00 & 0,24 & 2,61 & 0,00 & 0,47 & 0,00 & 0,71 & 0,00 & 0,24 & 0,71 \\
\hline $370-390$ & 79,66 & 2,74 & 7,21 & 0,50 & 0,75 & 1,00 & 0,50 & 1,00 & 0,00 & 0,23 & 0,50 & 1,49 & 3,73 \\
\hline $378-12$ & 90,00 & 3,88 & 3,34 & 0,00 & 0,51 & 1,03 & 0,00 & 0,10 & 0,00 & 0,00 & 0,40 & 0,10 & 0,00 \\
\hline $378-7$ & 91,50 & 1,66 & 5,00 & 0,00 & 0,17 & 0,50 & 0,17 & 0,33 & 0,00 & 0,00 & 0,00 & 0,00 & 0,50 \\
\hline
\end{tabular}


Una aproximación petrográfica a los modos de hacer y la circulación alfarera...

\begin{tabular}{|c|c|c|c|c|c|c|c|c|c|c|c|c|c|}
\hline Muestra & $\mathbf{M z}$ & $\mathrm{Cv}$ & $\mathrm{Qz}$ & Fk & Pl & Bi & Mu & Af-Px & LM & LG & LS & LV & Vv \\
\hline $380-13$ & 92,18 & 3,18 & 3,50 & 0,00 & 0,10 & 0,32 & 0,10 & 0,10 & 0,00 & 0,00 & 0,00 & 0,10 & 0,32 \\
\hline $370-517$ & 93,94 & 2,59 & 2,27 & 0,10 & 0,10 & 0,10 & 0,10 & 0,10 & 0,00 & 0,30 & 0,00 & 0,00 & 0,30 \\
\hline $370-446$ & 71,65 & 3,54 & 11,65 & 0,51 & 0,25 & 2,78 & 0,76 & 0,51 & 0,00 & 0,00 & 0,00 & 0,00 & 7,09 \\
\hline $367-1$ & 79,58 & 3,29 & 5,86 & 0,00 & 2,58 & 0,47 & 0,00 & 0,95 & 0,00 & 0,00 & 0,00 & 4,46 & 0,70 \\
\hline $378-28$ & 78,29 & 2,82 & 7,44 & 0,20 & 0,80 & 2,01 & 0,00 & 0,80 & 0,00 & 0,40 & 0,00 & 0,00 & 2,21 \\
\hline $365-25$ & 80,24 & 3,77 & 7,54 & 0,70 & 0,70 & 3,06 & 0,70 & 0,94 & 0,00 & 0,00 & 0,00 & 0,00 & 1,18 \\
\hline 560-T3 & 81,71 & 4,26 & 10,23 & 0,28 & 0,85 & 0,57 & 0,85 & 0,10 & 0,00 & 0,00 & 0,00 & 0,10 & 0,10 \\
\hline 584-4 & 77,87 & 5,80 & 12,57 & 0,00 & 0,82 & 0,55 & 1,37 & 0,27 & 0,00 & 0,00 & 0,00 & 0,55 & 0,00 \\
\hline $553-1$ & 87,12 & 1,67 & 5,02 & 1,00 & 1,17 & 0,67 & 0,50 & 0,00 & 0,00 & 2,85 & 0,00 & 0,00 & 0,00 \\
\hline $566-1$ & 81,80 & 3,36 & 9,80 & 0,56 & 0,56 & 1,40 & 0,28 & 0,56 & 0,00 & 0,56 & 0,00 & 0,00 & 0,84 \\
\hline 552-T1 & 74,78 & 3,93 & 9,88 & 1,27 & 0,76 & 0,13 & 0,25 & 0,13 & 1,39 & 4,44 & 0,00 & 1,77 & 0,13 \\
\hline 587-T6 & 62,50 & 6,16 & 14,21 & 2,05 & 2,57 & 4,46 & 0,86 & 0,17 & 0,00 & 4,97 & 0,00 & 0,00 & 0,00 \\
\hline 587-T1 & 65,20 & 6,08 & 14,25 & 1,05 & 3,98 & 2,31 & 0,42 & 0,84 & 1,67 & 1,89 & 0,00 & 1,05 & 0,00 \\
\hline $442-1$ & 72,29 & 4,34 & 6,75 & 2,41 & 3,61 & 0,72 & 0,00 & 0,00 & 0,00 & 8,67 & 0,00 & 0,00 & 0,00 \\
\hline $449-16$ & 63,01 & 6,24 & 6,24 & 1,50 & 1,72 & 3,24 & 0,00 & 0,00 & 0,00 & 15,05 & 0,00 & 0,00 & 0,43 \\
\hline 454-6 & 67,37 & 8,12 & 9,56 & 1,45 & 1,45 & 0,41 & 0,22 & 0,00 & 0,00 & 4,16 & 0,00 & 0,00 & 0,41 \\
\hline $448-17$ & 69,38 & 4,20 & 13,58 & 2,96 & 1,23 & 1,73 & 0,24 & 0,00 & 0,00 & 5,68 & 0,00 & 0,00 & 0,50 \\
\hline $443-2$ & 60,24 & 4,95 & 10,85 & 1,41 & 3,07 & 4,48 & 0,24 & 0,00 & 0,00 & 11,08 & 0,94 & 0,00 & 0,00 \\
\hline $448-18$ & 84,19 & 3,55 & 3,55 & 0,00 & 0,64 & 0,97 & 0,97 & 0,00 & 0,00 & 0,64 & 0,32 & 0,00 & 3,56 \\
\hline $448-14$ & 80,90 & 1,57 & 7,59 & 0,52 & 0,52 & 1,31 & 1,05 & 0,00 & 0,00 & 0,78 & 0,00 & 0,00 & 1,57 \\
\hline $448-15$ & 73,47 & 3,69 & 10,32 & 0,98 & 0,74 & 0,24 & 0,50 & 0,25 & 0,00 & 0,98 & 0,24 & 0,00 & 6,39 \\
\hline $442-3$ & 77,94 & 5,97 & 5,79 & 0,54 & 0,72 & 1,45 & 0,18 & 0,18 & 0,00 & 1,08 & 1,45 & 0,18 & 0,00 \\
\hline $447-20$ & 88,23 & 3,92 & 2,20 & 0,50 & 0,24 & 0,24 & 0,24 & 0,25 & 0,00 & 0,50 & 0,00 & 0,98 & 1,72 \\
\hline $454-1$ & 91,46 & 1,58 & 3,78 & 0,00 & 0,63 & 0,95 & 0,32 & 0,32 & 0,00 & 0,00 & 0,00 & 0,32 & 0,32 \\
\hline $444-7$ & 92,22 & 2,28 & 2,05 & 0,00 & 0,23 & 0,23 & 0,00 & 0,69 & 0,00 & 0,23 & 0,00 & 0,69 & 0,69 \\
\hline $454-4$ & 92,19 & 1,95 & 2,18 & 0,00 & 0,22 & 0,20 & 0,22 & 0,22 & 0,00 & 0,00 & 0,00 & 1,30 & 1,30 \\
\hline Laz113 & 92,88 & 2,56 & 3,01 & 0,10 & 0,31 & 0,10 & 0,10 & 0,10 & 0,00 & 0,21 & 0,00 & 0,00 & 0,53 \\
\hline Laz112 & 90,26 & 2,89 & 4,73 & 0,27 & 0,52 & 0,27 & 0,52 & 0,00 & 0,00 & 0,27 & 0,00 & 0,00 & 0,00 \\
\hline Corte 6 & 86,20 & 2,08 & 3,39 & 0,52 & 0,52 & 1,56 & 0,26 & 1,04 & 0,00 & 0,00 & 0,00 & 1,30 & 1,04 \\
\hline Corte 9 & 86,55 & 1,65 & 7,80 & 0,23 & 0,47 & 0,71 & 0,47 & 0,23 & 0,00 & 0,00 & 0,00 & 0,00 & 0,95 \\
\hline 31981 & 81,71 & 1,94 & 6,46 & 0,86 & 0,43 & 1,08 & 0,43 & 0,21 & 0,00 & 0,43 & 0,21 & 0,64 & 4,96 \\
\hline 33899 & 81,89 & 2,49 & 7,30 & 0,50 & 0,83 & 1,33 & 0,00 & 0,16 & 0,00 & 0,16 & 0,16 & 0,16 & 4,69 \\
\hline 31995 & 80,10 & 4,06 & 6,82 & 0,37 & 0,55 & 1,48 & 0,18 & 0,55 & 0,00 & 0,55 & 0,00 & 0,18 & 4,98 \\
\hline Corte 12 & 84,97 & 3,40 & 6,44 & 0,18 & 0,36 & 0,89 & 0,00 & 0,36 & 0,36 & 0,00 & 0,00 & 0,54 & 2,14 \\
\hline r377 & 53,33 & 6,67 & 11,75 & 4,56 & 4,74 & 5,79 & 1,05 & 0,53 & 0,00 & 0,53 & 0,00 & 1,58 & 9,47 \\
\hline v297 & 77,00 & 3,68 & 7,82 & 2,76 & 1,61 & 2,53 & 1,61 & 0,00 & 0,23 & 0,00 & 0,00 & 0,00 & 2,76 \\
\hline v458 & 87,35 & 4,08 & 2,04 & 0,81 & 0,82 & 0,63 & 0,60 & 0,00 & 0,00 & 0,00 & 0,00 & 0,00 & 3,67 \\
\hline Corte 7 & 80,24 & 3,34 & 7,38 & 0,47 & 0,95 & 0,95 & 0,24 & 0,95 & 0,00 & 1,43 & 1,20 & 0,95 & 1,19 \\
\hline Corte 13 & 75,24 & 4,25 & 13,15 & 0,77 & 0,58 & 2,13 & 1,55 & 0,39 & 0,58 & 0,00 & 0,00 & 0,39 & 0,39 \\
\hline 32083 & 93,43 & 2,28 & 2,47 & 0,38 & 0,19 & 0,38 & 0,19 & 0,10 & 0,00 & 0,10 & 0,00 & 0,00 & 0,38 \\
\hline 32108 & 78,09 & 5,40 & 11,22 & 0,98 & 0,39 & 0,98 & 0,39 & 0,59 & 0,00 & 0,19 & 0,00 & 0,10 & 2,75 \\
\hline 32192 & 87,04 & 1,87 & 5,19 & 0,86 & 0,29 & 0,58 & 0,14 & 0,14 & 0,00 & 0,72 & 0,00 & 0,29 & 2,16 \\
\hline 31907 & 90,03 & 3,57 & 3,24 & 0,34 & 0,17 & 0,51 & 0,17 & 0,17 & 0,00 & 0,10 & 0,00 & 0,17 & 1,36 \\
\hline 32214 & 76,00 & 4,80 & 10,60 & 0,20 & 0,20 & 1,60 & 0,80 & 0,40 & 0,00 & 0,60 & 0,00 & 0,40 & 3,60 \\
\hline 32185 & 68,83 & 4,33 & 13,20 & 1,51 & 0,86 & 1,29 & 0,21 & 0,65 & 0,00 & 0,21 & 0,00 & 0,43 & 8,27 \\
\hline 29375 & 71,77 & 5,18 & 11,66 & 1,19 & 1,29 & 1,29 & 1,29 & 0,64 & 0,00 & 1,07 & 0,00 & 0,64 & 3,45 \\
\hline v449 & 83,01 & 2,15 & 4,78 & 1,91 & 1,44 & 0,96 & 0,96 & 0,00 & 0,24 & 0,24 & 0,00 & 0,96 & 3,35 \\
\hline r108 & 77,20 & 5,87 & 3,67 & 1,23 & 2,70 & 1,23 & 1,72 & 0,25 & 0,49 & 0,00 & 0,00 & 0,98 & 4,66 \\
\hline v313 & 79,51 & 4,55 & 3,73 & 0,62 & 2,28 & 2,28 & 2,07 & 0,00 & 0,00 & 0,00 & 0,00 & 0,41 & 4,55 \\
\hline v37 & 63,26 & 4,30 & 4,97 & 1,66 & 8,94 & 3,64 & 1,32 & 0,33 & 0,33 & 0,00 & 0,00 & 1,32 & 9,93 \\
\hline $6(1) 473$ & 70,32 & 3,25 & 3,66 & 2,03 & 3,66 & 1,45 & 1,00 & 0,00 & 1,22 & 0,00 & 0,00 & 2,84 & 10,57 \\
\hline $6(1) 352$ & 79,92 & 6,42 & 3,21 & 2,00 & 2,41 & 0,81 & 0,81 & 0,00 & 0,00 & 0,00 & 0,00 & 0,40 & 4,02 \\
\hline V312 & 78,86 & 4,88 & 3,66 & 1,22 & 1,63 & 0,41 & 0,40 & 0,00 & 0,81 & 0,00 & 0,00 & 1,63 & 6,50 \\
\hline v131 & 62,33 & 6,88 & 8,44 & 4,69 & 3,75 & 4,69 & 0,31 & 0,00 & 0,00 & 7,50 & 0,00 & 0,16 & 0,00 \\
\hline r257 & 64,12 & 12,28 & 8,06 & 5,76 & 1,34 & 0,00 & 1,34 & 0,00 & 0,00 & 6,72 & 0,00 & 0,00 & 0,38 \\
\hline
\end{tabular}


Páginas anteriores, tabla 3. Porcentaje de cada uno de los elementos cuantificados para el análisis modal. Referencias: Mz (matriz), Cv (cavidades), Qz (cuarzo), Fk (feldespato potásico), $\mathrm{Pl}$ (plagioclasa), Bi (biotita), Mu (muscovita), Af-Px (anfíbol, piroxeno), LM (litoclasto metamórfico), LG (litoclasto granítico), LS (litoclasto sedimentario), LV (Litoclasto volcánico),

Vv (vidrio vesicular). Por su relativa escasez no se incluye en esta tabla los porcentajes correspondientes a microclino, turmalina, granate y minerales opacos.

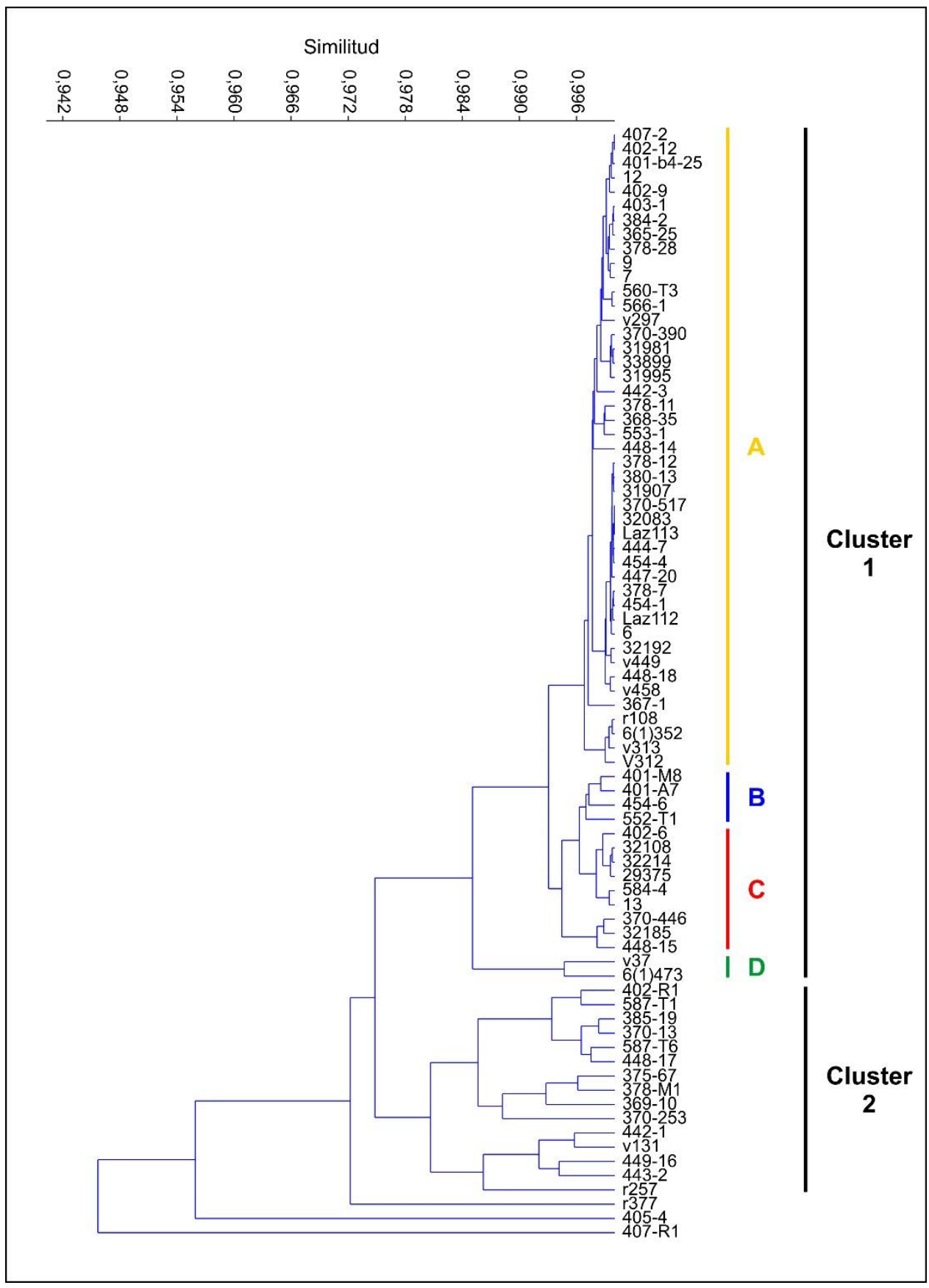

Figura 9. Dendrograma resultante del análisis composicional de la muestra. 
Luego, con las mismas variables utilizadas para el Análisis de Conglomerados se realizó un Análisis de Componentes Principales (ACP), del cual se obtuvieron tres componentes que explican el 93,61\% de la varianza total. El componente 1 expresa el 83,05\% de la variación y está dominado por la alta correlación positiva de la matriz $(0,92)$ y la correlación negativa del resto de las variables. El componente 2 representa el 6,10\% de la varianza y se asocia principalmente con la variable vidrio vesicular $(0,69)$ y en menor medida por plagioclasas $(0,17)$ y litoclastos volcánicos $(0,13)$. Además, se registra una correlación negativa de litoclastos graníticos $(-0,59)$, cuarzo $(-0,28)$ y matriz $(-0,19)$. Por último el componente 3 , define el $4,44 \%$ de la varianza total y presenta valores de correlación positiva de cuarzo $(0,78)$ y correlación negativa de litoclastos graníticos $(-0,54)$ y plagioclasas $(-0,20)$.

En la tabla 10 se observa el diagrama de dispersión del componente 1 vs 2 . Las muestras que se distribuyen en el cuadrante inferior izquierdo corresponden a pastas densas (con porcentaje de inclusiones mayor a $26 \%$ ), es decir, con alto porcentaje de inclusiones no plásticas principalmente representadas por cuarzo y clastos graníticos. Estos ejemplares corresponden a las modalidades medias M5, M6 y M7 y gruesa M8. En cambio, las muestras ubicadas en los cuadrantes superiores se explican por la abundancia relativa del vidrio vesicular en sus pastas, distribuyéndose gradualmente de izquierda a derecha según el porcentaje total del temperante registrado. Allí se encuentran distribuidos ejemplares de las modalidades finas (M2 y M3). Por su parte, las muestras que se hallan en el cuadrante inferior derecho corresponden a pastas poco densas (con porcentaje de inclusiones inferior a 16\%) (M2, M3 y M4), en las que predominan los clastos de cuarzo. Finalmente, en el extremo del cuadrante inferior derecho se ubican los casos con escasa cantidad de inclusiones no plásticas identificados bajo la modalidad fina M1.

Si comparamos los agrupamientos establecidos en el análisis de conglomerados y la distribución de las muestras en el ACP, observamos que el conjunto estudiado se diferencia por la combinación de la densidad y el tipo de antiplástico. De este modo, por un lado las pastas con mayor contenido granítico corresponden a las más densas, y por el otro, las menos densas están asociadas al predominio de temperante de origen volcánico. A su vez, las primeras son las pastas de modalidades gruesas y algunas medias, en cambio las otras, principalmente están asociadas a las modalidades de pastas finas.

A partir de esto cabe preguntarnos, ¿cómo se vinculan estos datos con la procedencia y las características estilísticas de las muestras? En este sentido, ¿cómo interpretar los resultados en relación con la circulación de piezas y conocimientos sobre cómo elaborarlas? Para ello, en primer lugar es necesario caracterizar la geología de la región de estudio y vincularla con los datos petrográficos de la cerámica. Luego, analizar las relaciones entre modalidades, composiciones y estilos entre los distintos valles. 
Figura 10. Gráfico de dispersión de los componentes 1 vs 2.

\section{Geología local y áreas de producción}

Las características geológicas de las regiones de hallazgo de los conjuntos estudiados, junto con la información obtenida a partir de análisis petrográficos de muestras de arenas ${ }^{1}$ han permitido proponer áreas de producción de las cerámicas en cuestión.

A partir de la asociación petrográfica entre las arenas estudiadas del sur Calchaquí y las inclusiones registradas en la cerámica arqueológica se postuló que gran parte del material alfarero analizado correspondería una producción de carácter local. El faldeo occidental del Aconquija está constituido por un ambiente de rocas ígneas intrusivas ácidas (granitos migmatíticos, granodiorita, etc.) propias del batolito granítico que caracteriza el área (González Bonorino 1951), y de cuya meteorización se originan las arenas examinadas, coincidiendo éstas - por la abundancia de cristales de cuarzo y fragmentos de roca graníticas- con las inclusiones registradas en las pastas cerámicas de los sitios Morro de las Espinillas, Tesoro I y Loma Alta (Pereyra Domingorena 2010, 2013).

Por otro lado, el río Santa María ha modelado un ancho valle con una llanura aluvional rica en sedimentos areno- limosos, cuya litología está enmarcada por los procesos orogénicos que dieron lugar a la formación de las sierras de Quilmes y las Cumbres 
Calchaquíes. En sí mismo, el valle presenta formaciones del Grupo Santa María, representado por areniscas, limonitas, arcillitas y conglomerados. En tanto que el fondo de la cuenca está formado por todos los depósitos producto del acarreo sedimentario del río Santa María durante el cuaternario (Ruiz Huidobro 1972). Las inclusiones registradas (granito, esquisto y arenisca) en la cerámica arqueológica se asemejan entonces a los materiales arenosos - de granulometría y composición muy variada-que son esperables en los sedimentos de una cuenca aluvional como la del río Santa María (Pereyra Domingorena 2010, 2013). Tal asociación material es propia de las acciones erosivas que afectaron las Cumbres Calchaquíes y la sierra del Cajón, de las cuales provendrían el material granítico y el esquisto de las pastas cerámicas del sitio Bañado Viejo; en tanto la arenisca debe proceder de las rocas sedimentarias de origen continental que integran el Grupo Santa María del Terciario, formación ubicada al este del yacimiento antes mencionado (Ruiz Huidobro 1972; Galván 1981).

Finalmente, también se ha podido detectar que algunas piezas presentaban una relativa abundancia de inclusiones de vidrio vesicular y litoclastos volcánicos, lo cual permitió postular que quizá esas vasijas habían sido elaboradas en el valle de Hualfín (Pereyra Domingorena 2010) por las similitudes registradas en la cerámica del área (Zagorodny y Balesta 2005).

Respecto a la cerámica Aguada recuperada en el sitio Los Viscos, valle de El Bolsón, a partir de los estudios composicionales y tecnológicos realizados previamente se propuso que esas vasijas no fueron elaboradas en la zona sino que posiblemente circularon desde el valle de Hualfín (Puente 2017). En estas pastas se reconoce el predominio de inclusiones de vidrio vesicular y otros clastos volcánicos, materias primas abundantes en ambas regiones. Sin embargo, las piezas de este estilo son excepcionales en el valle de El Bolsón y en cambio, en Hualfín predominan para la época.

Ambos valles, están interconectados por un mismo curso de agua cuyo nombre cambia a lo largo del recorrido. Concretamente, el río El Bolsón, nace en la falda Occidental de Chango Real, sigue naturalmente hacia el sur donde pasa a denominarse río Villa Vil y luego, a la altura de la localidad de El Eje intercepta al río Los Nacimientos -que corre al E de las sierras de Hualfín-, y pasan a conformar el río Belén. En El Bolsón, predominan dos formaciones litológicas de origen terciario -El Áspero y El Cajón- constituidas por un conjunto de sedimentos continentales con intercalación de origen volcánico (González Bonorino 1950; Turner 1973). La Formación El Áspero se manifiesta en el valle por una facie lávica formada por brechas (andesitas de color verde azulado, con numerosos fenocristales de anfíbol y plagioclasas) y tobas de composición básica y vitroclástica de tipo ácido (Turner 1973). Sobre ella se superpone la Formación El Cajón, en la que abundan areniscas arcósicas 
gruesas, conformadas principalmente por material granítico (puede ser migmatítico), con rodados de pizarra cuarzosa, gneiss milonítico, micacitas y andesita. El sedimento limoso micáceo y con vidrio, funciona como cemento. Se observan bancos de material tufítico y otros de material tobáceo. Estos últimos integrados por vidrio, plagioclasas, cuarzo y micas, entre otros (González Bonorino 1950: 45; Turner 1973: 34-35). El río El Bolsón acarrea de N a S material correspondiente a las formaciones El Cajón, Loma Corral y Chango Real. La Formación Loma Corral está integrada por rocas metamórficas sin aporte magmático, principalmente esquistos, pizarras y filitas. Por su parte, la Formación Chango Real está compuesta por granitos migmatíticos de tipo homogéneo y con anatexitas. Además, los cauces de ríos y arroyos temporarios, tributarios del río El Bolsón acarrean tanto desde el E como desde el O material de la formación El Áspero y de la Formación El Cajón (Turner 1973). Los estudios petrográficos realizados sobre láminas delgadas de muestras de arenas tomadas en distintos puntos que atraviesa el río en los sectores medio y sur del valle, evidencian la combinación de litoclastos volcánicos, metamórficos y graníticos, vidrio vesicular, cuarzo, plagioclasas y feldespato potásico, entre otros. Además, a pocos metros del sitio Los Viscos, en la ladera oeste de la sierra de Las Cuevas, se detectaron depósitos de arena integrados por más del 90\% de vidrio vesicular (Puente 2012).

Estos componentes observados en las arenas de El Bolsón también están presentes en el valle de Hualfín. Las rocas graníticas y metamórficas conforman el basamento cristalino sobre el cual se apoya una secuencia sedimentaria, compuesta por sedimentos clásticos continentales con intercalación de sedimentitas volcániclasticas y rocas andesíticas y basálticas de las formaciones El Cajón y El Morterito (González Bonorino 1950; Turner 1973; Zagorodny et al. 2010; Iucci 2013). Los análisis disponibles sobre petrografía de arena de la zona corresponden a muestras del cauce del río Corral Quemado -afluente del río Hualfín/Belén- y evidencian la combinación de fragmentos pumíceos (vidrio vesicular), cuarzo, feldespato potásico, plagioclasas y clastos líticos plutónicos, volcánicos y en menor medida metamórficos y sedimentarios. De este modo, se destacan considerables semejanzas en la composición de los sedimentos de uso potencial como temperante cerámico entre Hualfín y El Bolsón.

Por último, la petrografía de las vasijas ordinarias de Los Viscos evidencia diferencias con la geología regional y con la petrografía cerámica de muestras locales del periodo Tardío. Por ello se propone que se elaboraron con materias primas de otras zonas pero hasta el momento se desconoce su procedencia (Puente 2017). 


\section{Petrografía, modalidades y estilos: discutiendo la circulación de piezas y conocimientos}

Integrar la información petrográfica generada de forma independiente para cada región, posibilita analizar la relación entre modalidades, composiciones y estilos desde una escala macrorregional y de este modo, problematizar la circulación y procedencia de piezas y saberes sobre cómo elaborarlas.

Las piezas elaboradas con gredas sin agregado de temperante -M1- no permiten establecer inferencias en torno al área de manufactura ni de procedencia de materias primas, debido a que las inclusiones naturales presentes en las arcillas utilizadas no son diagnósticas por su escasez y tamaño pequeño. Específicamente corresponden a 10 ejemplares Aguada Pintado, Aguada Grabado y Pulido Fino, recuperados en sitios al sur del valle Calchaquí y a tres Aguada Grabado hallados en la localidad de la Ciénaga, valle de Hualfín. Para determinar las áreas de manufactura/procedencia de esta modalidad se necesita integrar estos resultados a los que se obtengan por análisis de activación neutrónica intrumental (AANI) y de ablación láser acoplada a espectrometría de masas con fuente de plasma de acoplamiento inductivo (LA-ICP-MS).

En las vasijas manufacturadas con las modalidades de pastas finas M2 y M3 (muestras recuperadas en el valle de El Bolsón, Hualfín y el sur Calchaquí,) y la media M4 (el sur Calchaquí) se observan dos tendencias en su composición petrográfica: una de ellas con vidrio vesicular y en menor medida con clastos volcánicos, la otra con mayor contenido de cuarzo y con escasez o ausencia de vidrio. La primera se asocia a las materias primas identificadas en los valles de El Bolsón y Hualfín, y se observa en piezas de estilo Aguada Grabado, Aguada Pintado y Pulido Fino, mientras que la segunda corresponde a una producción local al sur del valle Calchaquí y se reconocen también en vasijas de esos estilos. Sin embargo, los cuencos 370-446, 367-1 y 378-28 (Pulido Fino), la escudilla 402-6 (Pulido Fino), la tinaja 448-15 (Aguada Pintado) y el fragmento 370-390 (Aguada Pintado) recuperados en el sur Calchaquí poseen una composición petrográfica que se asocia a la litología de Hualfín y a la descripción petrográfica de piezas que se consideran de manufactura local en dicho valle (Baldini et al. 2005; Zagarodny y Balesta 2005). A partir de esto se infiere que estos ejemplares llegaron al sur Calchaquí como bienes manufacturados o se elaboraron con materias primas de Hualfín o aledañas. De este modo, se evidencia la circulación de bienes o recursos de una región hacia la otra. Además, en ambas hay producción local de piezas con las modalidades M2 y M3, sostenemos que esto es consecuencia de conocimientos compartidos entre los alfareros. Estos resultados apoyan la propuesta de la circulación de saberes a escala macrorregional planteada previamente desde los recursos estéticos y plásticos (Scatollin 2007a). Sin embargo, los datos disponibles no permiten determinar cómo funcionaron esos circuitos de interacción. 
Las siete piezas manufacturadas con la modalidad media M5 fueron elaboradas con antiplásticos principalmente de origen plutónico, como litoclastos graníticos y cuarzo. Específicamente, cinco corresponden a vasijas halladas en el sur de los valles Calchaquíes tres piezas ordinarias, una Baño Blanco y una Candelaria Acanalado- y se las considera de manufactura local (Pereyra Domingorena 2010). En cambio, las dos recuperadas en el sitio Los Viscos, valle de El Bolsón, son de tipo ordinario y se consideran alóctonas al lugar de hallazgo. Como no tenemos información sobre la producción ordinaria de la zona del valle de Hualfín no podemos afirmar que estas ollas hayan acompañado a las vasijas Aguada que se consideran de esa zona.

Los ejemplares elaborados con la modalidad M6 -dos ordinarios y uno Baño Blancofueron hallados únicamente en el sitio Bañado Viejo, valle de Santa María. Su petrografía es consistente con las arenas de la zona. La única vasija asignada a la modalidad media M7 corresponde a una olla ordinaria recuperada en el sitio Tesoro I, en la Falda occidental del Aconquija. Todos estos recipientes son considerados de manufactura local por los componentes observados en sus pastas (Pereyra Domingorena 2010).

Con respecto a las piezas elaboradas con la modalidad gruesa M8, la composición petrográfica de sus pastas es con alto contenido granítico. Se trata de nueve ejemplares ordinarios hallados al sur de los valles Calchaquíes que fueron manufacturados localmente (Pereyra Domingorena 2010).

Finalmente, la modalidad M9 representada hasta el momento por una sola vasija ordinaria recuperada en el sitio Bañado Viejo presenta en su pasta litoclastos volcánicos y tiesto molido. Es una pasta atípica a las registradas en las modalidades petrográficas del sur Calchaquí.

En síntesis, los resultados alcanzados permiten reconocer que en los últimos siglos del primer milenio D.C. las sociedades aldeanas elaboraron piezas a partir de ideas estéticas y técnicas compartidas a escalas regionales amplias. Asimismo, se identificó la circulación de ejemplares elaborados entre los valles aquí analizados.

\section{Conclusiones}

Este trabajo permitió demostrar que las alfarerías grabadas, pintadas y/o pulidas, estéticamente similares de finales del primer milenio D.C., fueron manufacturadas en el sur Calchaquí y en el valle de Hualfín. También, algunos ejemplares circularon desde Hualfín hacia el sur Calchaquí y el valle de El Bolsón. Esto último, se infiere por la abundancia relativa de vidrio vesicular y otros clastos volcánicos presentes en las pastas de vasijas 
halladas en estas dos áreas, comparables a la cerámica producida en Hualfín. Si bien el conjunto estudiado no lo muestra, no descartamos que haya habido piezas elaboradas en el sur Calchaquí y en el valle de El Bolsón que hayan circulado hacia Hualfín, como ha sido evidenciado para cronologías anteriores y para material lítico (Lazzari et al. 2017). Del mismo modo, queda pendiente determinar el intercambio de piezas entre el valle de El Bolsón y el sur Calchaquí.

En síntesis, pudimos reconocer saberes técnicos compartidos, a través del registro de las mismas modalidades de pastas en las áreas geográficas estudiadas y la circulación de piezas a partir de las singularidades observadas en los temperantes. Sin embargo, entendemos que el estudio a futuro debería encararse a través de una ampliación de la muestra y la integración de la información generada de distintos análisis químicos (AANI y LA-ICP-MS). Es necesario incrementar la evidencia -vajilla de servicio y recipientes ordinarios- de El Bolsón así como las muestras toscas de Hualfín; y paralelamente, se debería extender e integrar el análisis a otras regiones del Noroeste argentino como son las Selvas Occidentales, otros valles de la región Valliserrana, la Puna catamarqueña y la llanura santiagueña. De esta manera, se logrará obtener una mirada integradora de la circulación de piezas y saberes involucrados en la producción de estas alfarerías particulares de finales del primer milenio D.C.

Agradecimientos: a las autoridades del Museo Etnográfico por el permiso otorgado para analizar materiales de su acervo. Este trabajo fue financiado por subsidios de la ANPCyT (PICT Raíces 2011 N 116, dirigido por M. Cristina Scattolin; PICT Jóvenes Investigares 2011 $\mathrm{N}^{\mathrm{o}}$ 260, dirigido por Lucas Pereyra Domingorena; PICT Jóvenes Investigares $2011 \mathrm{~N}^{\mathrm{o}} 1657$, dirigido por Verónica Puente; PICT 2007 N 38129, dirigido por Alejandra Korstanje) y el CONICET (PIP 2012 No 486, dirigido por M. Cristina Scattolin; PIP 2005 Nº 5906, dirigido por Laura Quiroga). Nuestro reconocimiento a los evaluadores anónimos por los comentarios sugeridos que permitieron mejorar la calidad el manuscrito. No obstante, el contenido del mismo es nuestra responsabilidad.

\section{Notas}

1- Los cortes delgados de las arenas se realizaron sobre muestras consolidadas en resina y las observaciones se efectuaron con microscopio óptico de luz transmitida. 


\section{Bibliografía citada}

Aschero, C.

2007 Comentario. Mesa 1: Interacciones Surandinas. Aspectos económicos, políticos e ideológicos. En Sociedades Precolombinas Surandinas: Temporalidad, Interacción y Dinámica Cultural del NOA en el Ámbito de los Andes Centro-Sur, V. Williams, B. Ventura, A. Callegari y H. Yacobaccio (eds.), pp. 99-108. TANOA, Buenos Aires.

Adams, A; Mackenzie, W. y C. Guilford

1997 [1984] Atlas de Rocas Sedimentarias. Masson, Barcelona.

Ávila F. y V. Puente

2008 ¿Circulación de símbolos? Calabazas pirograbadas en el Tardío. La Zaranda de Ideas 4: 109-118.

Baldini, M.; Cremonte, M.; Botto, I. y M. Díaz

2005 De felinos, pastas y pigmentos. La cerámica de Choya 68 desde una perspectiva arqueométrica. En La Cultura de la Aguada y sus Expresiones Regionales, S. Martín y M. Gonaldi (eds.), pp. 87-105. EUDELAR, SECyT, Universidad Nacional de La Rioja, La Rioja.

\section{Bugliani, M.}

2007 Métodos de ordenación y técnicas de agrupamiento aplicadas a los recipientes cerámicos: las vasijas de los cementerios de La Falda Occidental de Aconquija. En Arqueometría Latinoamericana, O. Palacios, C. Vázquez, T. Palacios y E. Cabanillas (eds.), pp. 245-350. Comisión Nacional de Energía Atómica - Centro Atómico Constituyentes, San Martín.

2008 Consumo y Representación en el Sur de los Valles Calchaquíes (Noroeste Argentino). Los Conjuntos Cerámicos de las Aldeas del Primer Milenio. John \& Erica Hedges, Oxford.

Cigliano, E. (Director)

1960 Investigaciones arqueológicas en el Valle de Santa María. Publicación 4. Instituto de Antropología, Facultad de Filosofía y Letras. Universidad Nacional del Litoral, Rosario.

Cremonte, $\mathrm{M}$.

1996 Investigaciones Arqueológicas en la Quebrada de La Ciénega (Dpto. de Tafí, Tucumán). Tesis de doctorado. Facultad de Ciencias Naturales y Universidad Nacional de La Plata, La Plata.

2014 Yavi-Chicha and the Inka expansion: a petrographic approach. Antiquity 88: 1261-1274. 
Cremonte, M.; Botto, I.; Canafoglia, M.; Hocsman, S.; Rodríguez, M.; Aschero, C. y D. Gazzoli

2010 Una nueva tecnología en cazadores-recolectores transicionales de la Puna Argentina. Caracterización de un recipiente con fibra vegetal. En La Arqueometría en Argentina y Latinoamerica, S. Bertolino, R. Cattáneo y A. Izeta (eds.), pp. 43-48. Facultad de Filosofía y Humanidades, Córdoba.

Courtois, L.

1976 Examen au Microscope Pétrographique des Céramiques Archéologiques. Notes et Monographies Techniques Nº 8 . CNRS, Paris.

Druc, I. y Q. Gwyn

1998 From clay to pots: a petrographical analysis of ceramic production in the Callejón de Huaylas, North-Central Andes, Perú. Journal of Archaeological Science 25: 707-718.

Galván, A.

1981 Descripción geológica de la Hoja 10e, Cafayate. Boletín 177. Servicio Geológico Nacional. Buenos Aires.

García, L.

1997 El material cerámico de Tomayoc. Bulletin de l'Institut Francais d'Études Andines 26(2): 177-193.

González, A.

1964 La Cultura de La Aguada del N. O. Argentino. Revista del Instituto de Antropología II-III: 205-253.

1977 Arte Precolombino de la Argentina. Introducción a su Historia Cultural. Filmediciones Valero, Buenos Aires.

González Bonorino, F.

1951 Descripción geológica de la Hoja 12e, Aconquija. Boletín 75. Dirección Nacional de Minería, Buenos Aires.

Hammer, Ø.; Harper, D. y P. Ryan

2001 PAST: Palaeontological statistics software package for education and data analysis. Palaeontología Electrónica 4(1): 9. 
Iucci, M.

2013 Petrografía de la fracción arena en la cerámica Belén. Aportes desde el análisis composicional y textural. En Arqueometría Argentina, Estudios Pluridisciplinarios, M. Ramos, M. Lanza, V. Helfer, V. Pernicone, F. Bognanni, C. Landa, V. Aldazabal y M. Fernández (eds.), pp. 175-194. Aspha Ediciones, Buenos Aires.

Korstanje, $\mathrm{M}$.

2005 La Organización del Trabajo en torno a la Producción de Alimentos en Sociedades Agropastoriles Formativas (Provincia de Catamarca, República Argentina). Tesis de doctorado. Facultad de Ciencias Naturales e Instituto Miguel Lillo, Universidad Nacional de Tucumán.

2007 Territorios campesinos: producción, circulación y consumo en los valles altos. En Producción y Circulación Prehispánicas de Bienes en el Sur Andino, A. Nielsen, M. Rivolta, V. Seldes, M. Vázquez y P. Mercolli (eds.), pp. 191-224. Editorial Brujas, Córdoba.

Korstanje, M. y A. Würschmidt

1999 Producir y recolectar en los valles altos del NOA: "Los Viscos" como caso de estudio. En En los Tres Reinos: Prácticas de Recolección en el Cono Sur de América, C. Aschero, M. Korstanje y P. Vuoto (eds.), pp. 51-160. Instituto de Arqueología y Museo - Facultad de Ciencias Naturales e Instituto Miguel Lillo - Universidad Nacional de Tucumán, San Miguel de Tucumán.

Lanteri, A.; Margaría, C. y M. Cigliano

2005 Análisis multivariado: técnicas de agrupamientos. Árboles de distancias. En Sistemática Biológica: Fundamentos Teóricos y Ejercitaciones, A. Lanteri y M. Cigliano (eds.), pp. 93-122. EDULP, La Plata.

Lazzari, M.

2006 Traveling Things and the Production of Social Spaces: An Archaeological Study of Circulation and Value in NW Argentina. Ph. D. dissertation. Columbia University, New York.

Lazzari, M.; Pereyra Domingorena, L.; Stoner, W.; Scattolin, M.; Korstanje, M. y M. Glascock 2017 Compositional data supports decentralized model of production and circulation of artifacts in the pre-Columbian south-central Andes. PNAS 114(20): E3917-E3926.

Middleton, A. e I. Freestone

1991 Recent Developments in Ceramic Petrology. Ocasional Paper No 81. The British Museum, London. 
Nielsen, A.

2006 Estudios internodales e interacción interregional en los Andes Circumpuneños: teoría, método y ejemplos de aplicación. En Esferas de Interacción Prehistóricas y Fronteras Nacionales Modernas: Los Andes Sur Centrales, H. Lechtman (ed.), pp. 29-69. IEP-IAR, Lima.

Nuñez, L. y T. Dillehay

1979 Movilidad Giratoria, Armonía Social y Desarrollo en los Andes Meridionales: Patrones de Tráfico e Interacción Económica. Universidad Católica del Norte, Antofagasta.

Olivera, D.; Escola, P.; Elías, A.; Pérez, S.; Tchilinguirian, P.; Salminci, P.; Pérez, M.; Grana, L.; Grant, J.; Vidal, A.; Killian Galván, V. y P. Miranda

2015 El Formativo en la Puna meridional: de la opción productiva a las sociedades agropastoriles plenas. En Crónicas Materiales Precolombinas. Arqueología de los Primeros Poblados del Noroeste Argentino, M. Korstanje, M. Lazzari, M. Basile, M. Bugliani, V. Lema, L. Pereyra Domingorena y M. Quesada (eds.), pp. 663-691. Sociedad Argentina de Antropología, Buenos Aires.

Orton, C.; Tyers, P. y A. Vince

1997 La Cerámica en Arqueología. Critica, Barcelona.

Ownby, M.

2010 Canaanite Jars from Memphis as Evidence for Trade and Political Relationships in the Middle Bronze Age. Ph. D. dissertation. University of Cambridge.

Pereyra Domingorena, L.

2010 Manufacturas Alfareras de las Sociedades Aldeanas del Primer Milenio d.C. al Sur de los Valles Calchaquíes. Tesis de doctorado. Facultad de Filosofía y Letras, Universidad de Buenos Aires.

2013 Cerámica experimental con materias primas del sur de los valles Calchaquíes (Noroeste argentino). Intersecciones en Antropología 14: 239-250.

Puente, V.

2012 Lo que "oculta" el estilo: materias primas y modos de hacer en la alfarería Belén. Aportes desde la petrografía de conjuntos cerámicos del Valle del Bolsón (Belén, Catamarca, Argentina). Estudios Atacameños 43: 71-94.

2017 Conjuntos cerámicos en contextos de interacción. Análisis diacrónico de la alfarería del sitio Los Viscos (Valle del Bolsón, Belén, Catamarca). Arqueología 23(2): 11-33. 
Ruiz Huidobro, O.

1972 Descripción geológica de la Hoja 11e, Santa María. Boletín 134. Servicio Nacional Minero Geológico, Buenos Aires.

Scattolin, M.

1990 Dos asentamientos formativos al pie del Aconquija. El sitio Loma Alta. (Catamarca, Argentina). Gaceta Arqueológica Andina V(17): 85-100.

2006a De las comunidades aldeanas a los curacazgos en el Noroeste argentino. Boletin de arqueología PUCP 10: 357-398.

2006b Categoremas indígenas y designaciones arqueológicas en el Noroeste argentino prehispánico. Chungara 38: 181-192.

2007a Estilos como recursos en el Noroeste argentino. En Procesos Sociales Prehispanicos en el Sur Andino. La Vivienda, la Comunidad y el Terrotorio, A. Nielsen, M. Rivolta, V. Seldes, M. Vázquez y P. Mercolli (eds.), pp. 291-321. Editorial Brujas, Córdoba.

2007b Santa María antes del año mil. Fechas y materiales para una historia cultural. En Sociedades Precolombinas Surandinas: Temporalidad, Interacción y Dinámica Cultural del NOA en el Ámbito de los Andes Centro-Sur, V. Williams, B. Ventura, A. Callegari y H. Yacobaccio (eds.), pp. 203-219. Instituto de Arqueología - FFyL - UBA, Buenos Aires.

2010 La organización del hábitat precalchaquí (500 a.C. - 1000 d.C.). En El Hábitat Prehispánico, M. Albeck, M. Scattolin y M. Korstanje (ed.), pp. 13-51. EdiUnJu, San Salvador de Jujuy.

Scattolin, M. y M. Albeck

$1994 \mathrm{El}$ asentamiento humano en la falda occidental del Aconquija (Catamarca, Argentina). Shincal 4: 35-65.

Scattolin, M.; Bugliani, M.; Izeta, A.; Pereyra Domingorena, L.; Lazzari, M. y L. Martínez 2001 Conjuntos materiales en dimensión temporal. El sitio Formativo "Bañado Viejo" (Valle de Santa María, Tucumán). Relaciones de la Sociedad Argentina de Antropología XXVI: 167-192.

Shennan, S.

1992 [1988] Arqueología Cuantitativa. Crítica. Barcelona.

Stoltman, J.

2001 The role of petrography in the study of archaeological ceramics. En Earth Sciences and Archaeology, P. Goldberg, V. Holliday y C. Reid Ferring (eds.), pp. 297-326. Kluwer Academic, Plenum Publishers, New York. 
Turner, J.

1973 Descripción geológica de la Hoja 11d, Laguna Blanca. Boletín 142. Servicio Nacional Minero Geológico, Buenos Aires.

Tarragó, M. y M. Scattolin

1999 La problemática del Período Formativo en el valle de Santa María. Actas del XII Congreso Nacional de Arqueología Argentina, tomo I, pp. 142-153. Universidad Nacional de La Plata, La Plata.

Zagorodny, N. y B. Balesta

2005 Estudio multidimensional de la alfarería de La Ciénaga. En Azampay: Presente y Pasado de un Pueblito Catamarqueño, M. Sempé, S. Salceda y M. Maffia (eds.), pp. 267-288. Ediciones Al Margen, La Plata.

Zagorodny, N; Morosi, M.; Iucci, M. y F. Wynveldt

2010 Estudios composicionales de las pastas de la cerámica tardía de distintos sitios del Valle de Hualfín (Belén, Catamarca). Arqueología 16: 125-149. 
